Discussion Paper No. 06-017

Selektivität und direkte Wirkungen von Vermittlungsgutscheinen:

Empirische Befunde aus der Einführungsphase

Friedhelm Pfeiffer und Henrik Winterhager

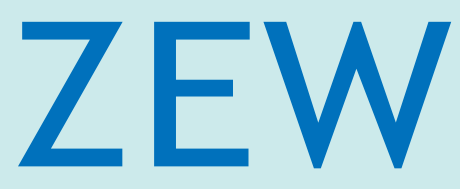

Zentrum für Europäische Wirtschaftsforschung $\mathrm{GmbH}$

Centre for European

Economic Research 
Discussion Paper No. 06-017

\section{Selektivität und direkte Wirkungen von Vermittlungsgutscheinen: Empirische Befunde aus der Einführungsphase}

Friedhelm Pfeiffer und Henrik Winterhager

Download this ZEW Discussion Paper from our ftp server:

ftp://ftp.zew.de/pub/zew-docs/dp/dp06017.pdf

Die Discussion Papers dienen einer möglichst schnellen Verbreitung von neueren Forschungsarbeiten des ZEW. Die Beiträge liegen in alleiniger Verantwortung der Autoren und stellen nicht notwendigerweise die Meinung des ZEW dar.

Discussion Papers are intended to make results of ZEW research promptly available to other economists in order to encourage discussion and suggestions for revisions. The authors are solely responsible for the contents which do not necessarily represent the opinion of the ZEW. 


\section{Das Wichtigste in Kürze}

Mit dem Vermittlungsgutschein werden private Vermittlungsaktivitäten öffentlich unterstützt. Ziel ist es, Arbeitslose wirksamer in Beschäftigung einzugliedern und die Vermittlungsprozesse zu beschleunigen. Die Probephase dieses im April 2002 eingeführten Instrumentes der aktiven Arbeitsmarktpolitik läuft noch bis zum Ende des Jahres 2006. Die vorliegende Studie untersucht Inanspruchnahme, direkte Wirkungen und Effizienz der VGS, die im Zeitraum Mai 2003 bis Juni 2004, also in der Einführungsphase, von Arbeitslosen in Anspruch genommen wurden. Die Wirksamkeit der VGS wird mit mikroökonometrischen, nicht-experimentellen Verfahren auf der Basis umfangreicher Prozessdaten der Bundesagentur abgeschätzt.

Der Verbreitungsgrad der VGS und vor allem ihr Beitrag zur Vermittlung von Arbeitslosen in Beschäftigung sind bisher quantitativ gering. Die direkten Wirkungen der VGS sind positiv; etwa 5 von je 100 Arbeitslosen, die einen VGS in Anspruch nahmen, gelangten den Schätzungen zufolge dadurch bedingt in Beschäftigung. In der Summe wurden damit mit den von Mai bis Dezember 2003 ausgegebenen VGS etwa 18.500 Arbeitslose ursächlich eingegliedert. Bei den übrigen 95 von 100 Arbeitslosen, die einen VGS in Anspruch nahmen, hat sich keine Veränderung ergeben. Der positive Effekt bezieht sich offensichtlich auf eine relativ kleine Gruppe von Arbeitslosen.

Neben den positiven direkten Wirkungen in der Gruppe der Arbeitslosen, die einen VGS in Anspruch nahmen, gibt es empirische Hinweise für negative indirekte Wirkungen in anderen Gruppen von Arbeitslosen. Auf der Basis der in der Arbeit vorgestellten Abschätzung von Kosten und Nutzen könnten diese bis zu 70 Prozent betragen, bevor der geschätzte Nettonutzen negativ wird. Die Ergebnisse der vorliegenden Untersuchung gelten nur für den beobachteten Zeitraum und konditional für die gleichzeitige Existenz der öffentlichen, unentgeltlichen Vermittlung.

Aus einer methodischen Sicht kann nach Ansicht der Autoren die hier zur Diskussion gestellte, relativ zeitnahe, Abschätzung der Wirkungen eines Programms der aktiven Arbeitsmarktpolitik mit Individualdaten wertvolle Informationen für eine rationale Weiterentwicklung der Arbeitsmarktpolitik bereitstellen. Jedoch darf der zeitliche und sonstige Aufwand für die Beschaffung der Daten und deren ökonometrischer Auswertung nicht unterschätzt werden. Für jedes Arbeitsmarktprogramm muss aufgrund seiner Besonderheiten idealerweise ein maßgeschneidertes Untersuchungsdesign entwickelt werden. Ohne eine Rückkopplung mittels mikroökonometrischer Evidenz besteht für die aktive Arbeitsmarktpolitik jedoch die Gefahr, nicht aus der Sackgasse herauszufinden. 


\title{
Selektivität und direkte Wirkungen von Vermittlungsgutscheinen: Empirische Befunde aus der Einführungsphase
}

\author{
Friedhelm Pfeiffer und Henrik Winterhager \\ Zentrum für Europäische Wirtschaftsforschung GmbH (ZEW) \\ L 7,1, D-68161 Mannheim
}

\section{Zusammenfassung:}

Die vorliegende Studie untersucht auf der Basis von Prozessdaten der Bundesagentur für Arbeit Inanspruchnahme, direkte Wirkungen und Effizienz von Vermittlungsgutscheinen, die im Zeitraum Mai 2003 bis Juni 2004, also in der Einführungsphase, von Arbeitslosen in Anspruch genommen wurden. Vermittlungsgutscheine haben in diesem Zeitraum eine relativ geringe Verbreitung gefunden. Für eine kleine Gruppe von 5 von 100 Arbeitslosen, die einen Vermittlungsgutschein in Anspruch nahmen, wird ein positiver direkter Beschäftigungseffekt ermittelt. Empirische Effizienzanalysen deuten darauf hin, dass der Nettonutzen positiv bleibt, wenn das Ausmaß indirekter negativer Wirkungen nicht mehr als 70 Prozent dieser Wirkungen übersteigt.

Schlüsselwörter: Arbeitsvermittlung, aktive Arbeitsmarktpolitik, Vermittlungsgutscheine, mikroökonometrische Evaluation

JEL-Klassifikation: J 68, H 25

Die Autoren danken dem Institut für Arbeitsmarkt- und Berufsforschung der Bundesanstalt für Arbeit (IAB), Nürnberg für die finanzielle Unterstützung (IAB-Projekt 544a, „Begleitforschung zur Vermittlung"), die die vorliegende Studie ermöglichte. Friedhelm Pfeiffer dankt ferner der Deutschen Forschungsgemeinschaft für die finanzielle Unterstützung im Rahmen der Projekte PF 331/2 und 3. Die Autoren möchten sich ferner bei Melanie Arntz, Kathrin Göggel, Anja Heinze, Thomas Kruppe, Gerrit Müller, Werner Sörgel, Alexander Spermann sowie einem anonymen Gutachter für wertvolle Anregungen zu einer früheren Version der Arbeit bedanken. Noch verbleibende Fehler und Unzulänglichkeiten gehen ausschließlich zu unseren Lasten. 


\section{$1 \quad$ Einführung}

Im August 1994 wurde das Vermittlungsmonopol der früheren Bundesanstalt für Arbeit gelockert (vgl. Konle-Seidl, 2002; Beschäftigungsförderungsgesetz, 1994). Private Arbeitsvermittler wurden zunächst in wenigen Marktsegmenten tätig, wie beispielsweise der Vermittlung von Künstlern (KonleSeidl und Walwei, 2002). Dies lag unter anderem daran, dass die Bundesagentur für Arbeit ihre Dienste Arbeitslosen und Unternehmen weiterhin ohne Entgelt anbot.

Eine weitergehende Förderung der privaten Vermittlung wurde mit der Einführung von Vermittlungsgutscheinen (VGS) in die Wege geleitet. Arbeitsuchende mit Anspruch auf Arbeitslosengeld oder -hilfe oder Teilnehmer an Arbeitsbeschaffungsmaßnahmen und Strukturanpassungsmaßnahmen, die nach einer Arbeitslosigkeit von drei Monaten noch nicht vermittelt sind, haben seit dem 1. April 2002 Anspruch auf einen VGS. ${ }^{1}$ Damit können sie einen privaten Vermittler ihrer Wahl einschalten, der im Falle einer erfolgreichen Vermittlung eine Vergütung von der zuständigen Agentur für Arbeit erhält. Die Höhe der Vergütung richtete sich bis Ende 2004 nach der Arbeitslosigkeitsdauer. ${ }^{2}$ Mit der Staffelung sollte nach dem Willen des Gesetzgebers dem Schwierigkeitsgrad der Vermittlung Rechnung getragen werden (vgl. Deutscher Bundestag, 2002).

Mit dem VGS soll die Vermittlung von Arbeitslosen in offene Arbeitsstellen sowie die Wiedereingliederung von Arbeitslosen beschleunigt werden. ${ }^{3} \mathrm{Ob}$ dieses Ziel tatsächlich erreicht wird, wird in den Agenturen für Arbeit und der Öffentlichkeit kontrovers diskutiert. Die Einführungsphase des VGS (bis Ende 2004) wurde im Rahmen eines vom IAB in Auftrag gegebenen Projek-

1 Sozialgesetzbuch $\S 421$ g SGB III; der VGS war zuletzt befristet bis Ende 2006. Vgl. auch IAB-Forum Nr. 1/06, Konle-Seidl (2005).

2 Sie belief sich nach einer Arbeitslosigkeit von bis zu 6 Monaten auf 1.500 Euro, nach 6 bis 9 Monaten auf 2.000 Euro und danach auf 2.500 Euro (seit dem Jahre 2005 gilt eine einheitliche Prämie von 2.000 Euro). Der private Vermittler erhält 1.000 Euro, falls das Beschäftigungsverhältnis mindestens drei Monate andauert, und den Rest dann, wenn das Beschäftigungsverhältnis sechs Monate oder länger währt. Um eine missbräuchliche Inanspruchnahme einzudämmen, gelten für die Auszahlung der Entgelte an die privaten Vermittler unter anderem folgende Regeln: zwischen Vermittler und Arbeitslosem muss ein schriftlicher Vermittlungsvertrag existieren, bei der vermittelten Arbeitsstelle handelt es sich um eine sozialversicherungspflichtige Beschäftigung mit einer Arbeitszeit von mindestens 15 Stunden wöchentlich, die vermittelte Arbeitsstelle gehört zu einem Unternehmen, bei dem der Arbeitslose im letzten Jahr vor der Arbeitslosmeldung nicht mehr als drei Monate lang versicherungspflichtig beschäftigt war.

3 Diese Ziele verfolgte die Bundesagentur für Arbeit bereits vor Einführung des VGS, beispielsweise durch die Förderung von gemeinnützigen Arbeitnehmerüberlassungsgesellschaften (vgl. Almus et al., 1998). 
tes „Begleitforschung zur Vermittlung“ wissenschaftlich untersucht. ${ }^{4}$ In dieser Arbeit werden ausgewählte mikroökonometrische Ergebnisse aus diesem Projekt vorgestellt und aus ökonomischer Sicht interpretiert. Weiterhin wird der potenzielle Beitrag einer wissenschaftlichen Begleitforschung für die Gestaltung der Arbeitsmarktpolitik am Beispiel des VGS diskutiert. Den Autoren standen für die Untersuchungen Prozessdaten der Bundesagentur für Arbeit zur Verfügung, insbesondere die Bewerberangebotsdatei (BewA), die Dateien der Integrierten Erwerbsbiografien (IEB) sowie VGS-spezifische Nutzungsdaten.

Ökonomische Studien zu den Determinanten von Vermittlungsprozessen zwischen Arbeitsuchenden und offenen Stellen legen den Schluss nahe, dass das individuelle Suchverhalten sowie die resultierenden Suchgleichgewichte durch Arbeitsrecht und die Arbeitsmarktpolitik beeinflusst werden können. Ein bedeutender Anteil der Unterschiede in der Fluktuation der Beschäftigung zwischen den USA und Kontinentaleuropa wird unter anderem mit Unterschieden im gesetzlichen Kündigungsschutz, mit Lohnrigiditäten und Transferleistungen in Verbindung gebracht, die Kosten und Nutzen von Suchprozessen beeinflussen. ${ }^{5}$

Aus theoretischer Sicht könnten VGS durchaus zur Beschleunigung der Suchprozesse beitragen, etwa wenn Arbeitsuchende oder Unternehmen durch private Vermittler Informationskosten über offene Stellen oder Bewerbereigenschaften sparen könnten. Insofern ließen sich grundsätzlich positive direkte Wirkungen erwarten, wobei jedoch aus betriebswirtschaftlicher Sicht umstritten ist, inwiefern die tatsächliche Ausgestaltung der VGS hinreichende Anreize für private Vermittler ausüben (Beckmann et al., 2004), ganz abgesehen von allgemeinen Gleichgewichtseffekten. So müssten denkbare positive direkte Effekte aus volkswirtschaftlicher Sicht relativiert werden, wenn sie nur eine Substitution der rein privaten oder rein öffentlichen Vermittlung, die ja weiterhin existiert, durch VGS widerspiegeln.

Obwohl die quantitativ bescheidene Inanspruchnahme von VGS in der Einführungsphase (siehe zu den Zahlen weiter unten) diese Skepsis zu rechtfertigen scheint, können zur Abschätzung der Wirkungen von Arbeitsmarktprogrammen ergänzende mikroökonometrische Analysen aufschlussreich sein. Die Literatur zur Evaluation aktiver Arbeitsmarktpolitiken legt die Vermutung nahe, dass auch Programme, deren gesamtwirtschaftliche Wirksamkeit

\footnotetext{
IAB-Projekt 544a „Begleitforschung zur Vermittlung - Implementation und Evaluierung von Beauftragungen nach § 37(a) SGB III, Vermittlungsgutscheinen und PSA“, vgl. auch Kruppe (2006).

5 Vgl. Franz und Pfeiffer (2005), Pfeiffer (2003), van der Linden (2005), und Pries und Rogerson (2005).
} 
umstritten ist, für bestimmte Gruppen von Arbeitslosen durchaus positive Wirkungen haben können (Heckman et al., 1999). Solche positiven direkten Wirkungen wurden beispielsweise für die gemeinnützigen Arbeitnehmerüberlassungsgesellschaften in Rheinland-Pfalz gefunden, bei gleichzeitiger Skepsis hinsichtlich der volkswirtschaftlichen Wirkungen (Lechner et al., 2001). Weitere mikroökonometrische Evidenz zur Wirksamkeit arbeitsmarktpolitischer Instrumente, die dem VGS vergleichbar sind, ist den Autoren nicht bekannt.

In der vorliegenden Studie wird die Wirksamkeit der Inanspruchnahme von VGS in den Jahren 2003 und 2004 mit dem Ansatz potenzieller Ergebnisse von Donald Rubin (Rubin, 1974, vgl. zur kritischen Würdigung und weiteren Anwendungen Lechner und Pfeiffer, 2001) abgeschätzt. Als direkte Wirkungen werden erstens der Beschäftigungserfolg bis zu 12 Monate nach der Aushändigung eines Gutscheins an einen Arbeitslosen sowie zweitens die dadurch induzierten Kosten-Nutzen-Relationen aus der Perspektive der Bundesagentur für Arbeit exemplarisch untersucht. Indirekte negative (Neben-)Wirkungen werden thematisiert, ebenso wie die relative Wirksamkeit des VGS im Vergleich zu Beauftragungen Dritter mit der gesamten Vermittlung, die zeitgleich mit dem VGS eingeführt wurden.

Die wichtigsten inhaltlichen Ergebnisse der Studie sind: VGS spielen in der hier betrachteten Einführungsphase für die Vermittlung von Arbeitslosen in Beschäftigung quantitativ keine bedeutsame Rolle; ihr Verbreitungsgrad ist nicht sehr hoch. Nur etwa 20 (37) Prozent der Arbeitslosen in den alten (neuen) Bundesländern, die im Jahr 2004 einen Rechtsanspruch hatten, nahmen einen VGS in Anspruch. Dies liegt unter anderem daran, dass die Bundesagentur für Arbeit weiterhin ihre Vermittlungstätigkeit unentgeltlich anbietet. Die direkten Wirkungen der VGS sind positiv. Etwa 5 von je 100 Arbeitslosen, die einen VGS in Anspruch nahmen, waren dadurch bedingt 12 Monate später in Beschäftigung. Weitere Analysen deuten darauf hin, dass negative indirekte (Neben-)Wirkungen existieren und dass bei vergleichbaren Arbeitslosen der VGS bessere Ergebnisse zeigte als Beauftragungen Dritter.

Die Arbeit ist wie folgt aufgebaut: In Abschnitt 2 werden mögliche Wirkungen des VGS aus theoretischer Sicht skizziert. Abschnitt 3 erläutert den Untersuchungsansatz der potenziellen Ergebnisse, Abschnitt 4 die verwendeten Prozessdaten der Bundesagentur für Arbeit. Abschnitt 5 diskutiert die ökonometrischen Befunde in Bezug auf Inanspruchnahme und Wirkungen des VGS. Abschließende Bemerkungen folgen in Abschnitt 6. 


\section{Wirkungen aus ökonomischer Sicht}

Das Ziel der VGS besteht darin, Arbeitslose schneller und nachhaltiger in eine reguläre Beschäftigung zu integrieren (Effektivität) und dies zudem kostengünstig zu erreichen (Effizienz). Aus theoretischer Sicht wird das Erreichen des Ziels Effektivität unter anderem von folgenden Bedingungen abhängen (siehe auch Beckmann et al., 2004; Ullrich, 2004):

1. Private Vermittler arbeiten möglicherweise effektiver als staatliche, sei es, dass letztere weitere Aufgaben neben der Vermittlung zu erfüllen haben, oder sei es, dass die unmittelbaren monetären Anreize bei ersteren höher sind. Hierbei gilt es zu bedenken, dass die staatlichen Agenturen ein umfassendes elektronisches Informationssystem vorhalten und möglicherweise aufgrund ihres langjährigen Erfahrungswissens Vorteile haben. Dieses Informationssystem steht jedoch auch den privaten Vermittlern (und den Arbeitslosen) ohne spezifisches Entgelt zur Verfügung. Im Ergebnis stellt der Weg über den privaten Vermittler eine Option für Arbeitslose dar, deren Nutzung in der Regel keine negative Auswirkung auf die Vermittlungseffektivität für diesen Arbeitslosen haben sollte.

2. Die Höhe des Entgelts für die Vermittlung wird als Steuerungsinstrument Art und Anzahl der Vermittelten pro Vermittler, wie auch das Marktsegment der privaten Vermittlung bestimmen. Private Vermittler werden ihre Aktivitäten soweit ausdehnen, dass das Entgelt für die marginale Vermittlung kostendeckend ist. Dabei gilt es zu bedenken, dass die VGS probeweise eingeführt wurden und die Unsicherheit über die Dauer deren Existenz eventuelle private Investitionen in die Vermittlungstätigkeit beeinflussen mag.

3. Das Instrument muss von den Arbeitslosen akzeptiert werden, da ein Vertrag zwischen dem Arbeitslosem und dem Vermittler Grundlage der Auszahlung des VGS ist. Arbeitslose müssen also bereit sein, mit einem privaten Vermittler zusammenzuarbeiten.

4. Schließlich stellen die Vermittlungsaktivitäten der Agenturen für Arbeit sowie deren sonstige Programme der aktiven Arbeitsmarktpolitik einen wichtigen institutionellen Rahmen dar, in dem sich das neue, zusätzliche Instrument VGS „,bewähren“ muss.

Arbeitslose haben Zugang zu einer Reihe von Weiterbildungs- und Vermittlungsmaßnahmen. Um den Rechtsanspruch auf einen VGS zu nutzen, müssen Arbeitsuchende über die Existenz des Instruments VGS informiert sein, seine Funktion verstehen, eine Vorstellung über entsprechende private Vermittler und deren Qualität oder Vertrauenswürdigkeit haben. Ferner sollten sie eine 
Vorstellung über die Vorteilhaftigkeit des VGS entwickeln, und zwar sowohl relativ zu anderen Instrumenten der aktiven Arbeitsmarktspolitik, relativ zu einer rein privaten Vermittlung wie auch relativ zu den eigenen Anstrengungen.

Die Gruppe der Arbeitslosen, die einen VGS in Anspruch nehmen, wird sich aufgrund dieser Selektion in ihren Eigenschaften von den übrigen unterscheiden. Auf individueller Ebene kann zumindest für diese Gruppe der Nutzer eine positive Beschäftigungswirkung erwartet werden. Das bedeutet jedoch noch nicht zwangsläufig, dass sich die öffentliche und private Vermittlungstätigkeit in der Summe verbessern wird. Vielmehr kann es sich um eine Verlagerung von öffentlicher auf private oder von der rein privaten auf die öffentlich geförderte Vermittlung handeln, ohne dass tatsächlich zusätzliche Vermittlungen zustande kommen. Insofern kann der VGS für die Gruppe der Arbeitslosen, die ihn nutzen, zwar positiv wirken, für einen zufällig ausgewählten Arbeitslosen allerdings keinen oder für Nichtnutzer sogar negative indirekte Effekte haben.

Das Wissen um die tatsächlichen Wirkungen für die verschiedenen Gruppen sowie eine fundierte Vorstellung von den Gesamtwirkungen ist zentral für eine rationale Fortentwicklung der Arbeitsmarktpolitik. Der in der vorliegenden Studie verwendete Forschungsansatz der potenziellen Ergebnisse von Donald Rubin kann bei Verwendung der Prozessdaten der Bundesagentur für Arbeit zu deren empirischer Fundierung und Fortschreibung beitragen.

Das zweite Ziel der VGS besteht in der Steigerung der Effizienz. Damit dieses Ziel erfüllt wird, müssten die privaten Vermittler, die sich über die Gutscheine finanzieren, kostengünstiger arbeiten als die staatlichen Agenturen, jedenfalls im Durchschnitt der vermittelten Arbeitslosen und bei gegebenem Zielerreichungsgrad. Als Kosten entstehen neben der Auszahlung der Gutscheine Verwaltungs- und Kontrollkosten. Aus theoretischer Sicht ist eine allgemeine Aussage dazu sicher kaum möglich. Im empirischen Teil der vorliegenden Untersuchung wird daher eine Methode zur empirischen Quantifizierung von Nutzen- und Kostengrößen zur Diskussion gestellt, die auf dem mikroökonometrischen Modell basiert. Im Rahmen der empirischen Effizienzanalyse wird untersucht, wie sich der Erhalt des Gutscheins auf die Höhe der im darauf folgenden Jahr von der Bundesagentur bezogenen Leistungen (Arbeitslosengeld und -hilfe, Unterhaltsgeld) auswirkt. Ferner werden Mehreinnahmen der Sozialversicherung und Steuern zumindest behelfsweise berücksichtigt. 


\section{Der Ansatz der potenziellen Ergebnisse}

Um die ursächlichen Wirkungen von Maßnahmen der aktiven Arbeitsmarktpolitik mit mikroökonometrischen Methoden abzuschätzen ${ }^{6}$, wird vielfach der Ansatz der potenziellen Ergebnisse verwendet. Bei diesem Ansatz wird davon ausgegangen, dass eine Zielvariable Y für eine Person zwei Ausprägungen annehmen kann, in Abhängigkeit davon, ob sie an einer Maßnahme teilgenommen hat: $Y_{1}$ bei Teilnahme und $Y_{0}$ bei Nichtteilnahme (potenzielle Ergebnisse). Es wird angenommen, dass diese Werte nicht davon abhängen, ob andere Personen teilnehmen oder nicht. Der direkte Maßnahmeeffekt für eine Person ist die Differenz der potenziellen Ergebnisse, $\mathrm{Y}_{1}-\mathrm{Y}_{0}$. Im Folgenden wird dargestellt, wie dieser Ansatz zur Analyse der Selektivität der Inanspruchnahme und der direkten Wirkungen von VGS eingesetzt wird.

Die direkte Wirkung des VGS wird aus der Differenz des tatsächlichen Wertes der Zielvariablen (Beschäftigung, Kosten-Nutzen-Relation) bei Inanspruchnahme eines VGS und des hypothetischen (oder kontrafaktischen, weil individuell nicht beobachtbaren) Wertes bei Nicht-Inanspruchnahme berechnet. Das grundlegende Evaluationsproblem besteht darin, den kontrafaktischen Zustand mit realen Daten abzuschätzen.

Dabei gilt es, wie im vorangehenden Abschnitt erörtert, zu berücksichtigen, dass die Inanspruchnahme eines VGS weder zufällig geschieht, noch von den Agenturen angeordnet werden kann. Diese Diskussion verdeutlichte zudem, dass außer dem VGS weitere Einflussfaktoren auf die Zielvariablen einwirken. In der nicht-experimentellen Evaluationsforschung wird mit ökonometrischen Verfahren der ursächliche Einfluss des VGS aus dem skizzierten Konglomerat von Faktoren herausgefiltert. Zu diesem Zweck wird in der vorliegenden Untersuchung ein Matching-Verfahren angewendet. Dabei wird jedem Arbeitslosen, der einen VGS in Anspruch nimmt, ein anderer Arbeitsloser zugeordnet, der keinen VGS in Anspruch nimmt und ansonsten in allen beobachteten persönlichen und agenturspezifischen Merkmalen nahezu identisch mit dem Ersteren ist.

Mit diesem Verfahren können bei Gültigkeit der zwei folgenden Annahmen die direkten (oder kausalen) Wirkungen der VGS abgeschätzt werden. Erstens handelt es sich dabei um die so genannte bedingte Unabhängigkeitsannahme. Diese ist theoretisch dann erfüllt, wenn bei Berücksichtigung aller relevanten beobachteten Merkmale (Selektion auf beobachtbare Variable) die

\footnotetext{
Siehe zur kritischen Würdigung der Methoden und weiteren Anwendungen beispielsweise Pfeiffer und Pohlmeier (1998), Lechner und Pfeiffer (2001).
} 
Höhe der Zielvariablen nicht von der Inanspruchnahme des VGS abhängt. Die zweite Annahme beinhaltet, dass es keine allgemeinen Gleichgewichtseffekte gibt. Die Vermittlung eines Arbeitslosen durch VGS würde demnach eine Arbeitsmarktlage voraussetzen, die derart ist, dass davon Vermittlungen von anderen Arbeitslosen nicht beeinflusst werden. Das wird tendenziell dann zutreffen, wenn es ständig viele offene Arbeitsstellen zu besetzen gibt, und wird dann eher nicht zutreffen, wenn Arbeitsmärkte eng sind. Auch die Gültigkeit der ersten Annahme hängt von der Art des Programms, den Eigenschaften der Arbeitslosen, der Arbeitsmarktlage, sowie Güte und Qualität der verwendeten Daten ab.

Ob diese beiden Annahmen für die Untersuchung tatsächlich erfüllt sind, bleibt eine Forschungsfrage. Es wurden Tests mit alternativen Spezifikationen, Stichproben und Variablen durchgeführt. Aufgrund dieser Tests, die wegen ihres Umfangs nicht im Detail dargestellt werden können, sind die folgenden Ergebnisse aus unserer Sicht robust, wenngleich Verzerrungen beispielsweise durch fehlende oder fehlerhafte Informationen letztlich nicht ausgeschlossen werden können.

\section{Datenbasis: Prozessdaten der Bundesagentur für Arbeit}

Die empirische Untersuchung basiert auf den prozessgenerierten Daten der Bundesagentur für Arbeit. ${ }^{7}$ Die Information, welcher Arbeitslose an welchem Tag einen Gutschein in welcher Höhe erhalten hat, wird in den Agenturen in coArb („computerunterstützte Arbeitsvermittlung“) festgehalten und anschließend in den so genannten Bewerberangebotsdaten (BewA) gespeichert. Die Einlösung von Gutscheinen durch private Vermittler sowie der Beginn des zugehörigen Beschäftigungsverhältnisses werden gesondert erfasst und mit den BewA wieder zusammengeführt.

Die Bewerberangebotsdaten sind nach Verlaufsdauern in Arbeitslosigkeit (Beginn und Ende einer Arbeitslosenperiode) geordnet, für alle registrierten Arbeitslosen verfügbar und enthalten sozio-demographische Merkmale sowie Informationen zur Dauer des letzten Beschäftigungsverhältnisses. Diesen Daten konnten für die Analyse der VGS zusätzlich Informationen zur Erwerbshistorie aus den Integrierten Erwerbsbiograhie Dateien (IEB, vgl. Hummel et

Vgl. auch Almus et al. (1998), Hujer et al. (2004), Hummel et al. (2005), Kruppe und Oertel (2003), Wilke und Winterhager (2004). 
al., 2005) des IAB zugespielt werden. ${ }^{8}$ Damit standen den Autoren sowohl Informationen zur Einmündung in Beschäftigung (Beginn, Ende, Entgelt) nach Inanspruchnahme eines VGS zur Verfügung, sowie über frühere Teilnahmen an Maßnahmen der aktiven Arbeitsmarktpolitik. ${ }^{9}$

Für die mikroökonometrische Abschätzung der potenziellen Ergebnisse durch Kontrollgruppenbildung („Matching“) wurden im Einzelnen folgende Informationen verwendet:

- sozio-demographische Merkmale: Geschlecht, Alter, Nationalität, Familienstand, Vorhandensein kleiner Kinder im Haushalt, Gesundheitszustand, Ausbildung,

- die Qualifikation und Erfahrung des Arbeitslosen aus der Sicht des Arbeitsvermittlers,

- gewünschte Arbeitszeit, gewünschter Beruf nach Angaben des Arbeitslosen,

- Beginn der aktuellen Arbeitslosigkeitsperiode, Höhe von Arbeitslosengeld bzw. -hilfe; Angaben zur letzten Beschäftigung: Berufsfeld, Tagesentgelt, Dauer und ob der Arbeitslose entlassen wurde, gekündigt hat oder einen befristeten Vertrag hatte,

- tagesgenaue Informationen zur Erwerbshistorie des Arbeitslosen in den vergangenen fünf Jahren, inklusive Angaben zu früheren Arbeitslosigkeitsperioden,

Agenturbezirk, in dem der Arbeitslose gemeldet ist; Arbeitslosenquote.

Da die Anzahl der zur Verfügung stehenden Merkmale groß ist, ist es in aller Regel und trotz großem Beobachtungsumfang nicht möglich, für jeden Arbeitslosen, der einen VGS in Anspruch nimmt, einen in allen Merkmalen gleichen anderen Arbeitslosen, der dies nicht tut, zu finden. ${ }^{10}$ Deshalb werden statistische Verfahren verwendet, mit denen Teilnehmer und Nichtteilnehmer

8 Auch die IEB basiert auf Verlaufsdauern, die vom IAB aus der Beschäftigtenhistorik (BeH), der Leistungsempfängerhistorikdatei (LeH) sowie der Maßnahme-Teilnehmer-Gesamtdatenbank (MTG) gespeist werden.

9 Die beschriebenen Daten wurden für die wissenschaftliche Untersuchung der VGS nach umfangreichen Aufbereitungen und Bereinigungen verwendet, die ausführlicher in Heinze et al. (2005) erläutert werden.

10 Man müsste beispielsweise für einen Arbeitslosen, der 35 Jahre alt und deutscher Staatsbürger ist, einen Hochschulabschluss, drei vorherige Arbeitslosigkeitsepisoden, im letzten Job 30.000 Euro pro Jahr verdient und körperliche Einschränkungen hat (usw.), einen entsprechenden Nichtteilnehmer findet. Während man bei stetigen Variablen (letztes Einkommen, Alter) noch zulassen kann, dass Kontrollpersonen nur ähnlich sind (34 Jahre alt, 31.000 Euro pro Jahr), ist dies bei kategorialen Variablen (Geschlecht, Staatsbürgerschaft) problematisch. 
auf Basis ihrer geschätzten Teilnahmewahrscheinlichkeit einander zugeordnet werden können (vgl. Rosenbaum and Rubin, 1983).

Die Ergebnisvariablen und über die Zeit variierende Merkmale müssen für die Matchingmethode in Relation zum Datum der Gutscheinausgabe definiert werden. ${ }^{11}$ Ein solches Datum ist für die Nichtbezieher nicht bestimmt. Daher wird jedem Nichtbezieher mit Rechtsanspruch auf einen VGS zufällig ein Datum zugeordnet, welches aus der diskreten Verteilung der Anfangsdaten der Bezieher gezogen wird (vgl. Lechner, 1999, 2002).

\section{Empirische Evidenz}

\subsection{Selektivität der Inanspruchnahme von VGS}

Abbildung 1 verdeutlicht die Inanspruchnahme von VGS seit der Einführung im April 2002 auf Monatsbasis. Nach einer eher zögerlichen Einführungsphase folgt eine stetige Zunahme, die sich jedoch seit April 2004 nicht mehr fortzusetzen scheint. Um eine Vorstellung von der Bedeutung der Inanspruchnahme von VGS zu erhalten, kann man diese Zahlen in Relation zu den anspruchsberechtigten Arbeitslosen setzen. Von allen Personen, die im Jahr 2004 einen Rechtsanspruch hatten, nahmen in den alten Bundesländern 20\% einen VGS in Anspruch, in den neuen 37\%. Im Jahre 2004 wurden 7,4 Prozent der ausgegebenen Vermittlungsgutscheine (etwa 54.000) innerhalb der Frist von drei Monaten eingelöst. Die überwiegende Anzahl der ausgegebenen VGS wurde demnach nicht eingelöst. Zu beachten ist, dass in der vorliegenden Untersuchung die Tatsache, dass ein Gutschein von der Agentur für Arbeit bezogen wird, als Maßnahme definiert wird.

Sowohl die Anzahl der Ausgaben wie auch der Einlösungen deutet auf ein Instrument der Vermittlung hin, dass in der Einführungsphase nur von einer quantitativ kleinen Gruppe von Arbeitslosen genutzt wurde (siehe auch Tabelle 1). Im Jahre 2004 wurden über 74 Millionen Euro an Prämien an private Vermittler ausgezahlt. Verglichen mit den 19,5 Mrd. Euro, die die Bundesagentur für Arbeit 2004 für aktive Arbeitsförderung ausgegeben hat, sind diese Ausgaben gering (vgl. Bundesagentur für Arbeit, 2004).

\footnotetext{
11 Eingliederung in reguläre Beschäftigung bis zu 12 Monate nach Ausgabe eines VGS, Dauer der Arbeitslosigkeit bis zur Gutscheinausgabe.
} 
Abbildung 1: Anzahl der ausgegebenen VGS pro Monat von April 2002 bis Dezember 2004

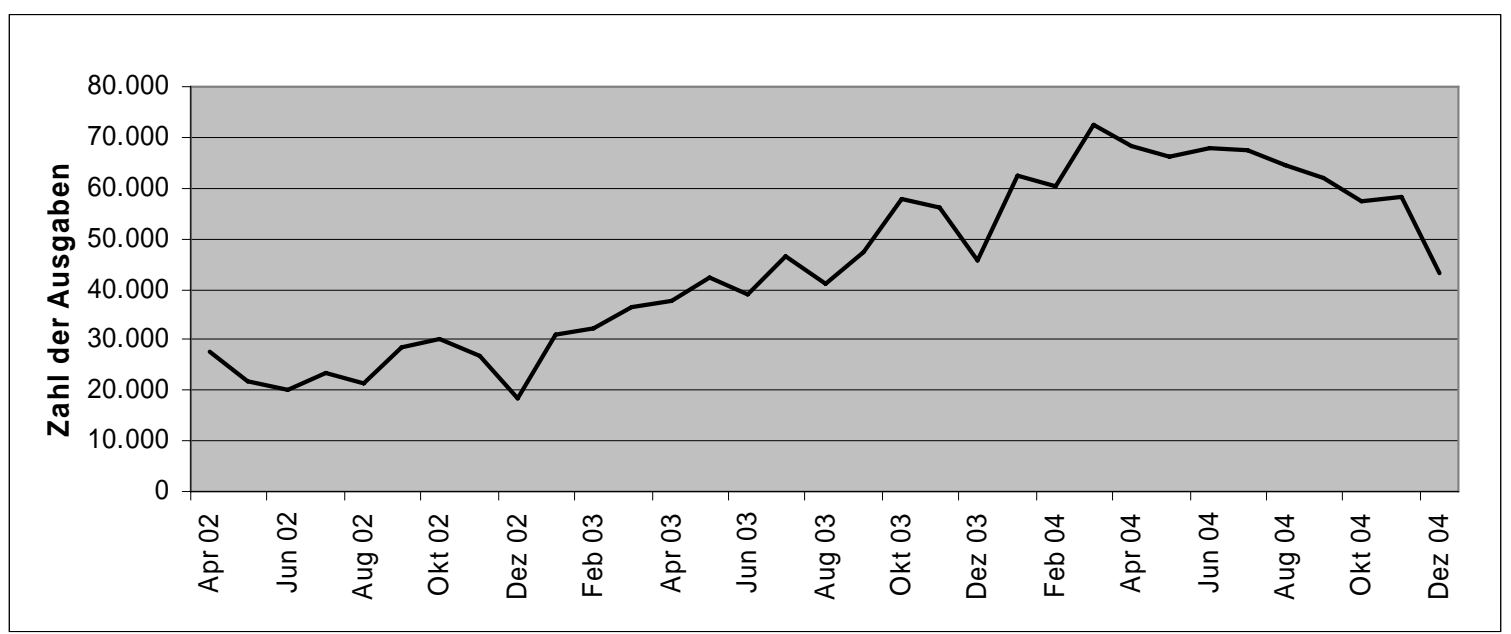

Quelle: Eigene Berechnungen mit den Daten der Bundesagentur für Arbeit, siehe Text.

Vergleicht man die Bezieher von VGS mit den Nichtbeziehern hinsichtlich ihrer individuellen Merkmale (Tabelle 1), so zeigt sich die Selektivität der Nutzung, die bei der Evaluation berücksichtigt werden muss. Für alle Variable mit einer Ausnahme ist der Unterschied zwischen Beziehern und Nichtbeziehern auf dem 1\%-Niveau signifikant (t-Test), bzw. für eine auf dem 5\%-Niveau signifikant. Die Bezieher sind im Durchschnitt jünger als die Nichtbezieher, weisen eine kürzere Arbeitslosendauer aus und sind seltener ohne Schulabschluss und Berufsausbildung. Ferner scheinen Frauen, Ausländer und Personen mit gesundheitlichen Problemen unterrepräsentiert zu sein. $^{12}$

In Abbildung 2 wird dargestellt, welcher Anteil der VGS-Bezieher und der Nichtbezieher ein Jahr vor und nach dem (hypothetischen) Ausgabezeitpunkt in regulärer Beschäftigung ist. Die Abbildung verdeutlicht, dass sowohl vor als auch nach dem Bezugszeitpunkt ein größerer Anteil von den VGS-Beziehern in Beschäftigung ist. Diese Unterschiede verdeutlichen die Selektivität bei der Gutscheinausgabe. Die Differenz in den Beschäftigungsanteilen nach der Ausgabe kann somit nicht allein auf die VGS zurückgeführt werden. ${ }^{13}$ Für Westdeutschland fällt zudem auf, dass bei beiden Gruppen der Anteil in Beschäftigung ein Jahr nach der Ausgabe noch weit unter dem Anteil ein Jahr vor der Ausgabe liegt.

12 Für die Bezieher und Nichtbezieher in 2004 ergibt sich ein quantitativ vergleichbares Bild. Die Ergebnisse sind auf Nachfrage erhältlich.

13 Für die VGS, die im ersten Halbjahr 2004 ausgegeben wurden, kann die Beschäftigung aufgrund der Datenlage nur für ein halbes Jahr nach der Ausgabe betrachtet werden. Für dieses halbe Jahr sowie vor der Ausgabe ergibt sich ein vergleichbares Bild wie für 2003. 
Tabelle 1: Arbeitslose, Anspruchsberechtigte und Bezieher von VGS sowie Vergleich von VGS-Beziehern und (anspruchsberechtigten) Nichtbeziehern anhand von ausgewählten Merkmalen (die Mittel- und Anteilswerte sind ein gewichtetes Mittel für die drei im oberen Teil der Tabelle genannten Zeiträume).

\begin{tabular}{|c|c|c|c|c|c|}
\hline & & \multicolumn{2}{|c|}{ Ost } & \multicolumn{2}{|c|}{ West } \\
\hline \multirow[t]{3}{*}{ Mai/Juni 2003} & Arbeitslose & \multicolumn{2}{|r|}{1.056 .252} & \multicolumn{2}{|r|}{2.429 .616} \\
\hline & Anspruchsberechtigte & \multicolumn{2}{|r|}{761.382} & \multicolumn{2}{|r|}{1.498 .962} \\
\hline & VGS-Bezieher & \multicolumn{2}{|r|}{33.220} & \multicolumn{2}{|r|}{39.253} \\
\hline \multirow[t]{3}{*}{ 3. Quartal 2003} & Arbeitslose & \multicolumn{2}{|r|}{1.085 .418} & \multicolumn{2}{|r|}{2.479 .211} \\
\hline & Anspruchsberechtigte & \multicolumn{2}{|r|}{748.697} & \multicolumn{2}{|r|}{1.473 .533} \\
\hline & VGS-Bezieher & \multicolumn{2}{|r|}{56.308} & \multicolumn{2}{|r|}{63.842} \\
\hline \multirow[t]{3}{*}{ 4. Quartal 2003} & Arbeitslose & \multicolumn{2}{|r|}{1.051 .804} & \multicolumn{2}{|r|}{2.464 .241} \\
\hline & Anspruchsberechtigte & \multicolumn{2}{|r|}{701.012} & \multicolumn{2}{|r|}{1.453 .573} \\
\hline & VGS-Bezieher & \multicolumn{2}{|r|}{51.569} & \multicolumn{2}{|r|}{88.127} \\
\hline & & $\begin{array}{l}V G S- \\
\text { Bezieher }\end{array}$ & $\begin{array}{l}\text { Nicht- } \\
\text { bezieher }\end{array}$ & $\begin{array}{l}\text { VGS- } \\
\text { Bezieher }\end{array}$ & $\begin{array}{l}\text { Nicht- } \\
\text { bezieher }\end{array}$ \\
\hline Alter in Jahren & & 38,93 & 41,99 & 38,58 & 40,49 \\
\hline Anteil der Fraue & & $41,64 \%$ & $51,08 \%$ & $35,64 \%$ & $38,77 \%$ \\
\hline Anteil der Auslä & Ider & $0,90 \%$ & $1,17 \%$ & $12,27 \%$ & $17,00 \%$ \\
\hline Gesundheitliche & Einschränkungen & $18,17 \%$ & $25,35 \%$ & $21,55 \%$ & $30,82 \%$ \\
\hline \multicolumn{6}{|c|}{ Dauer der Arbeitslosigkeit } \\
\hline bis zur Ausgabe & n Tagen ${ }^{\text {a) }}$ & 428,16 & 580,45 & 399,99 & 503,83 \\
\hline \multicolumn{6}{|c|}{ Schulabschluss (Anteile): } \\
\hline Kein Abschluss & & $5,70 \%$ & $8,92 \%$ & $10,58 \%$ & $15,67 \%$ \\
\hline Hauptschule & & $26,84 \%$ & $32,16 \%$ & $47,58 \%$ & $53,54 \%$ \\
\hline Mittlere Reife & & $60,45 \%$ & $53,09 \%$ & $27,21 \%$ & $19,12 \%$ \\
\hline Fachhochschulre & & $1,75 \%$ & $1,39 \%$ & $5,74 \%$ & $4,28 \%$ \\
\hline Abitur & & $5,26 \%$ & $4,45 \%$ & $8,89 \%$ & $7,39 \%$ \\
\hline \multicolumn{6}{|c|}{ Art der Berufsausbildung (Anteile): } \\
\hline \multicolumn{2}{|c|}{ keine Ausbildung } & $11,24 \%$ & $17,79 \%$ & $30,83 \%$ & $42,30 \%$ \\
\hline \multicolumn{2}{|c|}{ betriebliche Ausbildung } & $74,97 \%$ & $71,51 \%$ & $54,15 \%$ & $46,17 \%$ \\
\hline \multicolumn{2}{|c|}{ außerbetriebliche Ausbildung } & $5,70 \%$ & $3,37 \%$ & $3,86 \%$ & $2,19 \%$ \\
\hline Berufsfachschul & und Fachschule & $4,18 \%$ & $4,06 \%$ & $5,16 \%$ & $4,24 \%$ \\
\hline Hochschule/Uni & & $2,29 \%$ & $2,07 \%$ & $3,62 \%$ & $3,30 \%$ \\
\hline Fachhochschule & & $1,61 \%$ & $1,20 \%$ & $2,38 \%$ & $1,80 \%$ \\
\hline Berufserfahrung & & $82,11 \%$ & $84,30 \%$ & $86,12 \%$ & $87,36 \%$ \\
\hline Jobwechsel & & $41,93 \%$ & $35,27 \%$ & $49,40 \%$ & $48,89 \%$ \\
\hline Tagesentgelt der & letzten Beschäftigung (€) & 23,04 & 20,20 & 28,95 & 26,76 \\
\hline Leistungsbezug & ro Tag $(€)$ & 18,62 & 17,18 & 22,51 & 21,56 \\
\hline
\end{tabular}

Quelle: Eigene Berechnungen mit den Daten der Bundesagentur für Arbeit, siehe Text. 
Abbildung 2: Entwicklung des Anteils in reguläre Beschäftigung für VGSBezieher und (anspruchsberechtigte) Nichtbezieher ein Jahr vor und nach den Ausgabezeitpunkten (Ausgaben von Mai bis Dezember 2003)
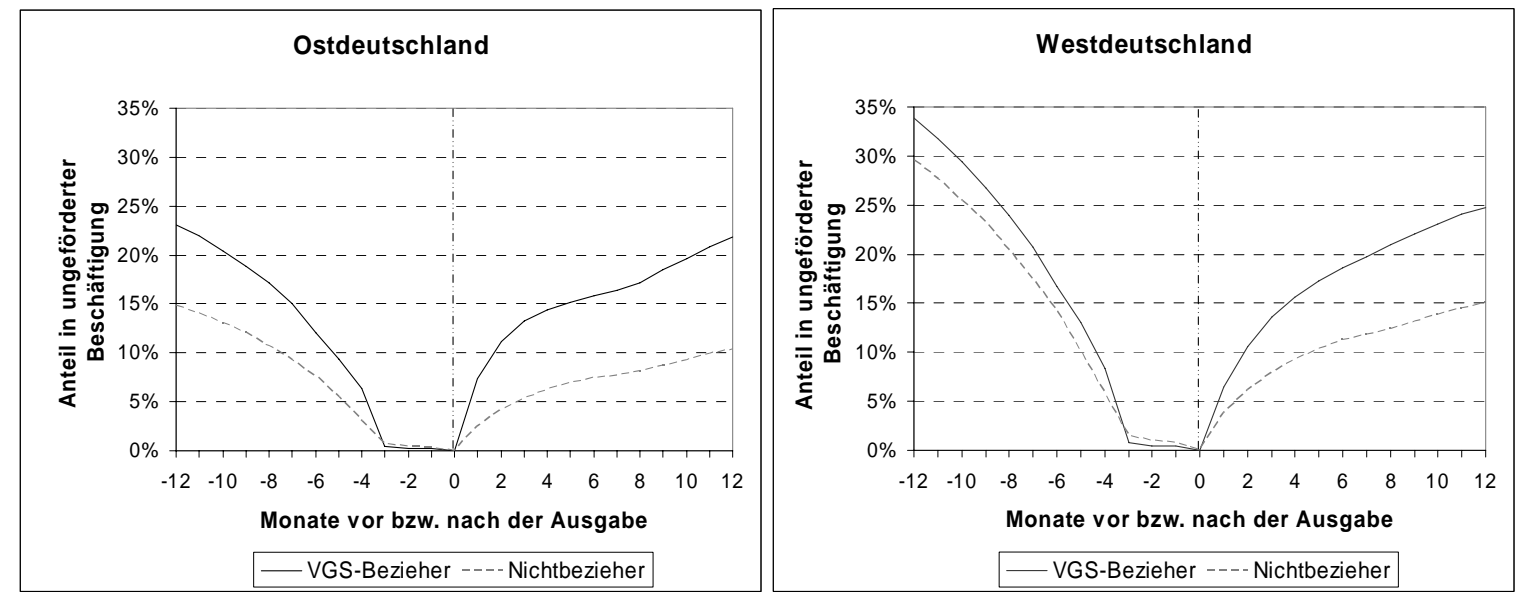

Quelle: Eigene Berechnungen mit den Daten der Bundesagentur für Arbeit, siehe Text.

Als weitere Zielvariable werden Kosten-Nutzen-Größen untersucht. Tabelle 2 fasst die Komponenten Summe der „Arbeitsverdienste“ und „Leistungen der Bundesagentur für Arbeit“ (berechnet als Durchschnittswert pro Person) innerhalb von 12 Monaten nach der Ausgabe des VGS zusammen. Während die VGS-Bezieher im Durchschnitt etwa doppelt so viel verdienen, weisen die Leistungen ein vergleichbares Niveau auf. Auch diese Ergebnisse bestätigen die Selektivität der Inanspruchnahme von VGS.

Tabelle 2: Arbeitsverdienst und Leistungsbezug pro Person in 12 Monaten nach (hypothetischer) Gutscheinausgabe für VGS-Bezieher und (anspruchsberechtigte) Nichtbezieher. Die Angaben beziehen sich auf die Ausgaben von Gutscheinen im Zeitraum Mai bis Dezember 2003.

\begin{tabular}{lcrlr}
\hline \hline & \multicolumn{2}{c}{ Ost } & \multicolumn{2}{c}{ West } \\
& $\begin{array}{l}\text { VGS- } \\
\text { Bezieher }\end{array}$ & $\begin{array}{l}\text { Nicht- } \\
\text { bezieher }\end{array}$ & $\begin{array}{l}\text { VGS- } \\
\text { Bezieher }\end{array}$ & $\begin{array}{l}\text { Nicht- } \\
\text { bezieher }\end{array}$ \\
\hline $\begin{array}{l}\text { Summe des Arbeitseinkommens } \\
\text { pro Person }(€)\end{array}$ & 3.457 & & & \\
$\begin{array}{l}\text { Summe des Leistungsbezugs pro } \\
\text { Person }(€)\end{array}$ & 4.656 & 3.702 & 2.083 \\
\hline \hline
\end{tabular}

Quelle: Eigene Berechnungen mit den Daten der Bundesagentur für Arbeit, siehe Text.

\subsection{Direkte Beschäftigungswirkungen von VGS}

Die Ergebnisse der Schätzungen des Propensity Scores durch ein Probit Modell sind im Anhang dargestellt, ebenso wie die Ergebnisse eines Balancing 
Tests zur Überprüfung der Qualität des Matching. Die mit MatchingVerfahren untersuchte erste Zielvariable ist eine sozialversicherungspflichtige Beschäftigung bis zu einem Zeitraum von 12 Monaten nach der Ausgabe des VGS, die nicht durch Maßnahmen der aktiven Arbeitsmarktpolitik gefördert wurde (im Folgenden reguläre Beschäftigung). Abbildung 3 zeigt die Anteile der Personen in Beschäftigung mit VGS sowie der Kontrollgruppe ein Jahr vor und ein Jahr nach der Gutscheinausgabe in monatlichen Abständen. ${ }^{14}$ Die Differenz der beiden Werte nach der Ausgabe ist die geschätzte (durchschnittliche) direkte Wirkung der VGS für die Gruppe der Bezieher von VGS.

Demnach waren beispielsweise von den VGS-Beziehern in den alten Bundesländern nach einem Jahr 24,8 Prozent in Beschäftigung. Ohne den Gutschein wären, bei Gültigkeit des empirischen Modells, nur 19,5 Prozent in Beschäftigung gewesen. Generell scheinen die Anteile der Arbeitslosen, die in Beschäftigung übergehen, mit weniger als 25 von Hundert gering zu sein. Dennoch verdeutlichen die mikroökonometrischen Analysen, dass VGS in den alten und den neuen Bundesländern signifikant (1-Prozent-Niveau) positive Effekte haben, die sich bereits in den ersten drei Monaten herausbilden. Der Durchschnittseffekt beträgt nach 12 (6) Monaten in den neuen Bundesländern 5,9 $(4,3)$ Prozentpunkte, in den alten 5,3 $(4,2)$.

Abbildung 3: Durchschnittliche direkte Effekte von VGS-Ausgaben Mai bis Dezember 2003; Entwicklung des Anteils in regulärer Beschäftigung für

VGS-Bezieher und Kontrollgruppe
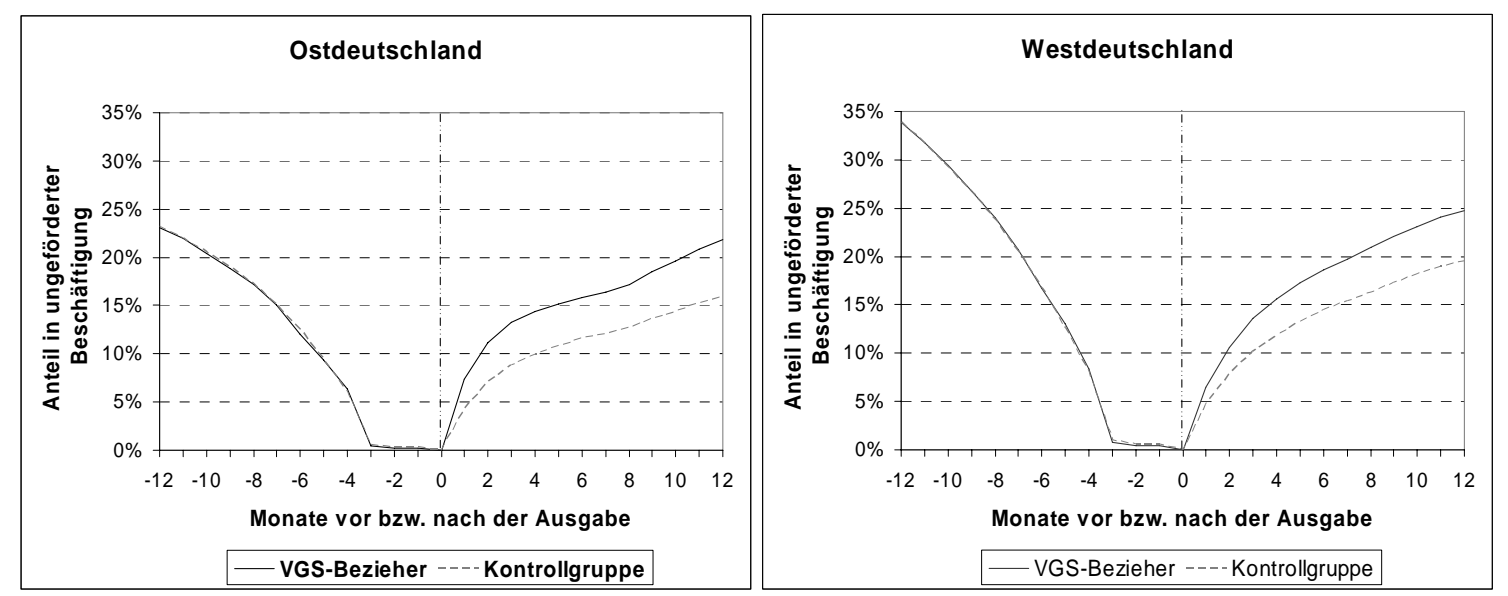

Quelle: Eigene Berechnungen mit den Daten der Bundesagentur für Arbeit, siehe Text; die geschätzten Effekte sind vom ersten Monat an auf dem 1\%-Niveau signifikant.

14 Die zugrunde liegenden Zahlen sowie die Standardfehler finden sich im Anhang. Dort wird auch erläutert, wie die Standardfehler berechnet wurden. 
Für die VGS, die im ersten Halbjahr 2004 ausgegeben wurden, kann die Wirkung aufgrund der Verfügbarkeit von geeigneten Daten nur für ein halbes Jahr betrachtet werden. Nach 6 Monaten beträgt der Beschäftigungseffekt 5,4 Prozentpunkte in den neuen und 5,1 in den alten Bundesländern. Diese Werte übersteigen geringfügig die entsprechenden Ergebnisse für das Jahr 2003. Dieser Unterschied liegt im Wesentlichen daran, dass verschiedene Jahreszeiten betrachtet wurden. Eine getrennte Berechnung nach Monaten hat gezeigt (nicht aus der Abbildung ersichtlich), dass ein saisonales Muster der Wirkungen existiert. Für die im 2. Quartal eines jeden Jahres ausgegebenen VGS werden signifikant höhere direkte Beschäftigungswirkungen gemessen. Private Vermittler scheinen demnach während der üblichen Frühjahrsbelebung erfolgreicher gewesen zu sein.

Die Höhe der durchschnittlichen Maßnahmeeffekte variiert in Abhängigkeit von der Entgeltstruktur des VGS und den soziodemographischen Merkmalen (Tabelle 3). Den empirischen Abschätzungen zufolge sind die Effekte bei Gutscheinen mit der höchsten Prämie am niedrigsten. Dies deutet darauf hin, dass der Anreiz zur Vermittlung von Langzeitarbeitslosen durch das höhere Entgelt nicht ausreicht, um die höheren Vermittlungskosten dieser Gruppe von Arbeitslosen zu kompensieren.

Bei der Differenzierung nach dem Geschlecht der Bezieher ergibt sich nur im Osten ein signifikanter Unterschied zugunsten der Männer. Bei der Differenzierung nach dem Alter zeigt sich, dass der Effekt bei jüngeren Arbeitslosen deutlich höher ausfällt als bei älteren. Das Altersprofil entspricht etwa dem Prämienprofil. Die Differenzierung nach der Berufsausbildung deutet darauf hin, dass der Effekt bei Personen mit betrieblicher Ausbildung, sowie in Westdeutschland für Fachhochschulabsolventen besonders hoch ist. 
Tabelle 3: Direkte durchschnittliche Effekte der VGS für deren Nutzer und die Zielvariable reguläre Beschäftigung 12 Monate nach der Ausgabe, differenziert nach Gutscheinwert und ausgewählten soziodemographischen Merkmalen (Ausgaben Mai bis Dezember 2003)

\begin{tabular}{lcc|cc}
\hline \hline & \multicolumn{2}{c}{ Ost } & \multicolumn{2}{c}{ West } \\
& $\begin{array}{c}\text { Durchschn. } \\
\text { Effekt }\end{array}$ & $\begin{array}{c}\text { Standard- } \\
\text { fehler }\end{array}$ & $\begin{array}{c}\text { Durchschn. } \\
\text { Effekt }\end{array}$ & $\begin{array}{c}\text { Standard- } \\
\text { fehler }\end{array}$ \\
\hline Gutscheinwert & & & & \\
VGS über 1.500 & $7,81 \%$ & $0,33 \%$ & $7,61 \%$ & $0,29 \%$ \\
VGS über 2.000 $€$ & $7,88 \%$ & $0,41 \%$ & $6,92 \%$ & $0,36 \%$ \\
VGS über 2.500 $€$ & $4,34 \%$ & $0,15 \%$ & $3,54 \%$ & $0,15 \%$ \\
\hline Geschlecht & & & & \\
Männer & $6,67 \%$ & $0,19 \%$ & $5,38 \%$ & $0,16 \%$ \\
Frauen & $4,71 \%$ & $0,21 \%$ & $5,27 \%$ & $0,22 \%$ \\
\hline Alter & & & & \\
Unter 25 Jahre & $8,74 \%$ & $0,46 \%$ & $7,43 \%$ & $0,47 \%$ \\
25 bis 50 Jahre & $5,90 \%$ & $0,17 \%$ & $5,45 \%$ & $0,16 \%$ \\
Über 50 Jahre & $3,64 \%$ & $0,29 \%$ & $3,63 \%$ & $0,28 \%$ \\
\hline Art der Berufsausbildung & & & & \\
keine Ausbildung & $4,75 \%$ & $0,34 \%$ & $4,57 \%$ & $0,22 \%$ \\
betriebliche Ausbildung & $7,18 \%$ & $0,66 \%$ & $6,67 \%$ & $0,74 \%$ \\
außerbetriebliche Ausbildung & $5,98 \%$ & $0,17 \%$ & $5,65 \%$ & $0,19 \%$ \\
Berufsfachschule und Fachschule & $2,68 \%$ & $0,69 \%$ & $5,98 \%$ & $0,61 \%$ \\
Hochschule & $4,98 \%$ & $0,96 \%$ & $4,12 \%$ & $0,72 \%$ \\
Fachhochschule & $4,48 \%$ & $1,17 \%$ & $6,78 \%$ & $0,91 \%$ \\
\hline \hline
\end{tabular}

Quelle: Eigene Berechnungen mit den Daten der Bundesagentur für Arbeit, siehe Text.

\subsection{Abschätzung von Kosten- und Nutzengrößen}

Die im Folgenden präsentierte Abschätzung von Kosten-Nutzen-Größen in Euro erhebt keinen Anspruch auf Vollständigkeit. Vielmehr soll exemplarisch das Potenzial der Analyse auch zur mikroökonometrischen Fundierung von Kosten-Nutzen-Größen von Arbeitsmarktprogrammen veranschaulicht werden. Der Einfluss von indirekten negativen Wirkungen (siehe dazu auch den folgenden Abschnitt) wird im Rahmen einer Sensitivitätsanalyse illustriert. Die Berechnungen basieren auf den zwischen Mai und Dezember 2003 ausgegebenen Gutscheinen. Die Kosten und Nutzen werden einerseits aus 
den Individualdaten, andererseits aus Informationen des internen Controllings der Bundesagentur für Arbeit abgeschätzt. Als Kosten berücksichtigen wir im Folgenden:

1. Prämienzahlungen für die eingelösten Gutscheine an die privaten Vermittler.

2. Verwaltungs- und Kontrollaufwand bei der Ausgabe und Einlösung von VGS in den einzelnen Arbeitsagenturen sowie anteilige Gemeinkosten. Die dem VGS zugerechneten Kosten wurden der Kosten- und Leistungsrechnung von 30 Agenturen für Arbeit entnommen und mittels eines Regressionsmodells für 180 Agenturen hochgerechnet.

3. Koordinationskosten auf Ebene der Regionaldirektionen und der Zentrale in Nürnberg. Einmalig entstandene Kosten für die Einführung neuer Software, Schulungen der Mitarbeiter oder die wissenschaftliche Evaluation bleiben unberücksichtigt.

Als Nutzengrößen werden berücksichtigt:

1. Einsparungen von Leistungen der BA durch die Wirkungen mittels der mikroökonometrischen Kontrollgruppen innerhalb von 12 Monaten nach Ausgabe.

2. Steuern und Sozialabgaben durch zusätzliche Beschäftigung mittels der mikroökonometrischen Kontrollgruppen innerhalb von 12 Monaten nach Ausgabe.

3. Weiter wurden die Einsparungen an Betreuungsaufwand in den Agenturen durch eine beschleunigte Vermittlung abgeschätzt, indem die geschätzte Reduzierung der Zahl der Arbeitslosenmonate durch die VGS mit einem kalkulatorischen Kostensatz bewertet wurde. Da sich die Kostenrechnung der Bundesagentur noch im Aufbau befindet, wurde dieser Kostensatz (vereinfacht) geschätzt, indem der Verwaltungsaufwand auf die Gesamtzahl der Arbeitslosenmonate umgelegt wurde.

Die Kosten-Nutzen-Abschätzung wurde für drei alternative Szenarien aufgestellt. Das Basisszenario (1) geht davon aus, dass der Effekt der Gutscheine nur für 12 Monate anhält, die in den Daten beobachtet werden, und danach gleich 0 ist. Bei Variante (2) wird angenommen, dass die Effekte zu 70 Prozent ein weiteres Jahr andauern und erst dann auf null sinken. Variante (3) unterstellt zusätzlich, dass die Effekte noch ein drittes Jahr anhalten, in Höhe von 40 Prozent des ersten Jahres. 
Tabelle 4: Ergebnisse der Kosten-Nutzen-Abschätzung für die VGS, die von Mai bis Dezember 2003 ausgegeben wurden

\begin{tabular}{|c|c|c|c|c|}
\hline $\begin{array}{l}\text { Nutzen des Instruments } \\
\text { im Szenario } 1\end{array}$ & \multicolumn{2}{|c|}{$\begin{array}{l}\text { Ostdeutschland } \\
75.956 .994\end{array}$} & \multicolumn{2}{|c|}{$\begin{array}{c}\text { Westdeutschland } \\
69.968 .153\end{array}$} \\
\hline \multirow[t]{2}{*}{ Kosten des Instruments } & \multicolumn{2}{|c|}{21.680 .666} & \multicolumn{2}{|c|}{ 19.512.198 } \\
\hline & Nettonutzen & $\begin{array}{l}\text { kritischer } \\
\text { Substitu- } \\
\text { tionseffekt }\end{array}$ & Nettonutzen & $\begin{array}{l}\text { kritischer } \\
\text { Substitu- } \\
\text { tionseffekt }\end{array}$ \\
\hline Szenario 1 & 54.276 .328 & $71,5 \%$ & 50.455 .954 & $72,1 \%$ \\
\hline Szenario 2 & 107.446.224 & $83,2 \%$ & 99.433 .661 & $83,6 \%$ \\
\hline Szenario 3 & 137.829 .022 & $86,4 \%$ & 127.420 .923 & $86,7 \%$ \\
\hline
\end{tabular}

Quelle: Eigene Berechnungen mit den Daten der Bundesagentur für Arbeit, siehe Text.

Für Ostdeutschland ergibt das Basisszenario (1) einen positiven Gesamtnettonutzen von 54,3 Mio. Euro (Tabelle 4). Für die anderen beiden Varianten ist der Nettonutzen wesentlich höher. Weiterhin wurde für jedes Szenario berechnet, bei welcher Höhe indirekter negativer Effekte gerade noch ein Nettonutzen von 0 erzielt würde. Dieser „kritische Substitutionseffekt“ gibt somit an, bis zu welcher Höhe von indirekten negativen Effekten das Instrument auch aus fiskalischer Sicht noch als effizient bezeichnet werden kann. Die Werte betragen je nach Szenario zwischen 71,5 und 86,2 Prozent. Für Westdeutschland beträgt der Nettonutzen in den drei Szenarien zwischen 50,5 und 127,4 Mio. Euro, der kritische Wert des Substitutionseffektes liegt zwischen 72,1 und 86,7 Prozent.

\subsection{Indirekte Beschäftigungswirkungen von Vermittlungsgut- scheinen}

Um die Existenz indirekter negativer Wirkungen der Ausgabe von VGS für Arbeitslose oder Beschäftigte, die keinen VGS genutzt haben, zu untersuchen, werden die Ergebnisse der als Teil der Begleitforschung durchgeführten makroökonometrischen Analysen (Hujer et al., 2006) herangezogen. Theoretisch können die positiven Wirkungen durch Verdrängungs- und Substitutionseffekte oder auch über den Finanzierungszusammenhang (die Mittel für VGS werden von den Beitragszahlern erbracht) relativiert werden. Nach den Ergebnisse von Hujer et al. (2006) hat die Anzahl von VGS keine signifikante Steigerung des Abgangs aus Arbeitslosigkeit oder der Höhe der Beschäftigung in den 180 Agenturen für Arbeit zur Folge. Das Fehlen des Nachweises eines makroökonomischen Impulses könnte für die Existenz be- 
deutsamer indirekter Wirkungen sprechen. Den positiven Wirkungen in der Gruppe der mit VGS geförderten Arbeitslosen könnten negative Wirkungen in nicht mit VGS geförderten Gruppen von Arbeitslosen gegenüberstehen. Dadurch, dass einige Arbeitslose schneller in Beschäftigung kamen, verzögerte sich die Eingliederung anderer.

\subsection{Weiterführende empirische Befunde}

Die Bundesagentur für Arbeit stellt für Arbeitslose eine ganze Reihe von Arbeitsmarktprogrammen zur Verfügung. Aus den bisherigen Untersuchungen wird noch nicht klar, wie die Größe des positiven Effektes in Relation zu anderen möglichen Programmen ausgefallen ist. Um darüber etwas in Erfahrung zu bringen, wurden im Rahmen des Projektes „Begleitforschung zur Vermittlung“ die direkten Wirkungen der Inanspruchnahme eines VGS relativ zu einem anderen Instrument der Vermittlung, den Beauftragungen Dritter mit der gesamten Vermittlung nach $\S 37$ SGB III ${ }^{15}$, abgeschätzt. Abbildung 5 zeigt die Ergebnisse des ökonometrischen Vergleichs der relativen Wirksamkeit der beiden Instrumente für das erste Halbjahr 2004 (entnommen aus Winterhager, 2006). Es wird deutlich, dass die Bezieher von VGS, die auch einem Dritten zur gesamten Vermittlung hätten zugewiesen werden können, in letzterem Fall schlechter abgeschnitten hätten.

Abbildung 5: Direkte Effekte von VGS-Ausgaben (erstes Halbjahr 2004) gegenüber der Alternative „Zuweisung zu einem Dritten zur gesamten Vermittlung“ (§ 37 SGB III); dargestellt wird die Entwicklung der Anteile in regulärer Beschäftigung
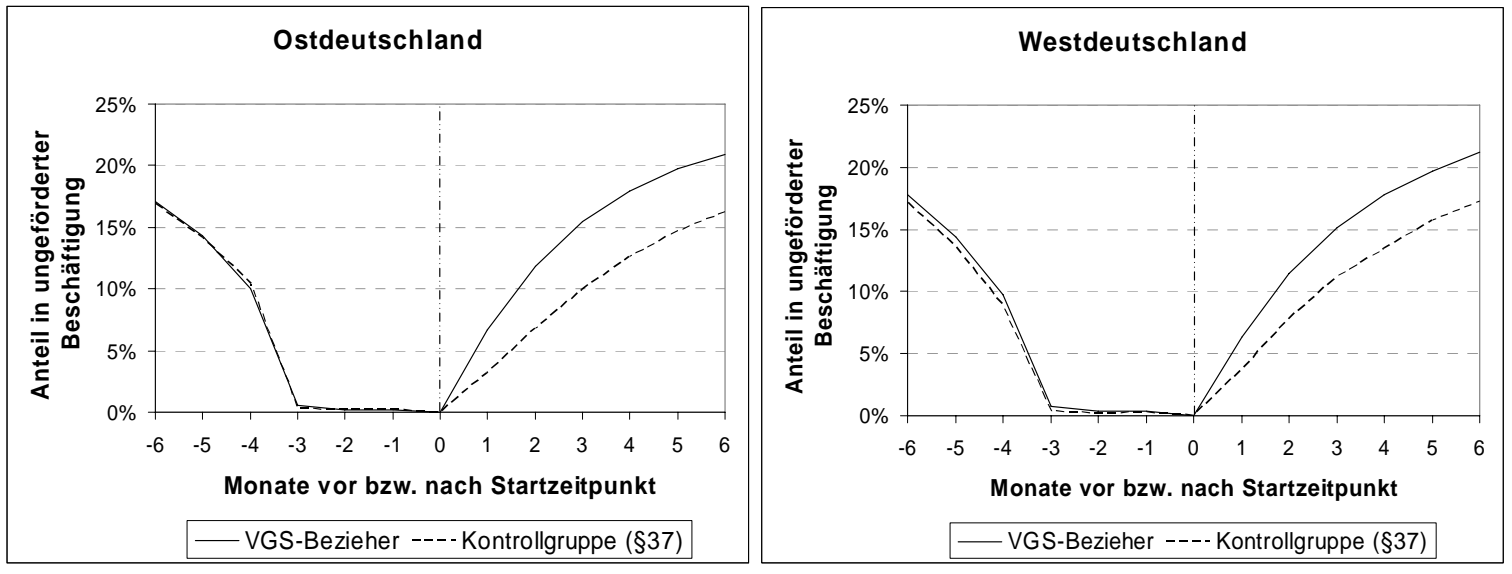

Quelle: Eigene Berechnungen mit den Daten der Bundesagentur für Arbeit; siehe Text; die geschätzten Effekte sind vom ersten Monat an auf dem 1\%-Niveau signifikant.

15 Kurz zusammengefasst schließt dabei die Agentur für Arbeit mit privaten Dritten Verträge über die Zuweisung eines bestimmten Kontingentes an Arbeitslosen ab. Anschließend werden den Dritten fortlaufend Arbeitslose zugewiesen, die in Beschäftigung zu vermitteln sind. Die Vergütung der Dritten besteht aus einem Erfolgshonorar für die Vermittlung sowie bei schwer zu vermittelnden Arbeitslosen aus einer Aufwandspauschale für die erfolgte Zuweisung. 
Der relative Effekt nach 6 Monaten beträgt 4,6 Prozentpunkte in den neuen und 4 Prozentpunkte in den alten Bundesländern und ist auf dem 1\%-Niveau signifikant. Somit waren im Jahre 2004 die Beauftragungen Dritter weniger gut geeignet als VGS, um Arbeitslose wieder in Beschäftigung zu bringen.

\section{Abschließende Bemerkungen}

Mit dem VGS werden private Vermittlungsaktivitäten öffentlich unterstützt. Ziel ist es, Arbeitslose wirksamer in Beschäftigung einzugliedern und die Vermittlungsprozesse zu beschleunigen. Die Probephase dieses im April 2002 eingeführten Instrumentes der aktiven Arbeitsmarktpolitik läuft noch bis zum Ende des Jahres 2006. Die vorliegende Studie untersucht Inanspruchnahme, direkte Wirkungen und Effizienz der VGS, die im Zeitraum Mai 2003 bis Juni 2004, also in der Einführungsphase, von Arbeitslosen in Anspruch genommen wurden. Die Wirksamkeit der VGS wird mit mikroökonometrischen, nicht-experimentellen Verfahren auf der Basis umfangreicher Prozessdaten der Bundesagentur abgeschätzt.

Der Verbreitungsgrad der VGS und vor allem ihr Beitrag zur Vermittlung von Arbeitslosen in Beschäftigung sind bisher quantitativ gering. Die direkten Wirkungen der VGS sind positiv; etwa 5 von je 100 Arbeitslosen, die einen VGS in Anspruch nahmen, gelangten den Schätzungen zufolge dadurch bedingt in Beschäftigung. In der Summe wurden damit mit den von Mai bis Dezember 2003 ausgegebenen VGS etwa 18.500 Arbeitslose ursächlich eingegliedert. Bei den übrigen 95 von 100 Arbeitslosen, die einen VGS in Anspruch nahmen, hat sich keine Veränderung ergeben. Der positive Effekt bezieht sich offensichtlich auf eine relativ kleine Gruppe von Arbeitslosen. Dies bestätigt im Allgemeinen die mikroökonometrische Evidenz zur Wirksamkeit anderer Programme der aktiven Arbeitsmarktpolitik (Heckman et al., 1999) und im Speziellen die Ergebnisse zur gemeinnützigen Arbeitnehmerüberlassung (Lechner et al., 2001).

Neben den positiven direkten Wirkungen in der Gruppe der Arbeitslosen, die einen VGS in Anspruch nahmen, gibt es empirische Hinweise für negative indirekte Wirkungen in anderen Gruppen von Arbeitslosen. Auf der Basis der in der Arbeit vorgestellten Abschätzung von Kosten und Nutzen könnten diese bis zu 70 Prozent betragen, bevor der geschätzte Nettonutzen negativ wird.

Beckmann et al. (2004) beurteilen die Chancen des VGS aufgrund betriebswirtschaftlicher, theoretischer Überlegungen pessimistisch. Sie begründen dies damit, dass erstens unklar ist, ob der Gutschein privaten Arbeitsvermittlern einen ausreichenden Anreiz für Aktivitäten insbesondere bei Problem- 
gruppen gibt, und dass zweitens die Arbeitslosigkeit gerade bei Problemgruppen vor allem struktureller Natur ist und nicht durch verbesserte Vermittlung behoben werden kann.

Die Ergebnisse der vorliegenden Untersuchung deuten darauf hin, dass der VGS in bestimmten Segmenten des Arbeitsmarktes für bestimmte, quantitativ kleine Gruppen positive Effekte in der Einführungsphase erzielt hat, und in dieser Hinsicht besser abgeschnitten hat als die Beauftragungen Dritter für die gesamte Vermittlung, die zur gleichen Zeit eingeführt wurden. Die relative Wirksamkeit zu sonstigen Instrumenten (z.B. geförderter Weiterbildung) wurde noch nicht untersucht. Nicht zuletzt sind jedoch die Anreize in der öffentlichen Vermittlung und das Zusammenspiel zwischen öffentlicher und privater Vermittlung noch zu wenig erforscht, um die Wirksamkeitsanalyse der VGS in der Einführungsphase abschließen zu können. Zudem gelten die hier vorgestellten Ergebnisse nur für den beobachteten Zeitraum und konditional für die gleichzeitige Existenz der öffentlichen, unentgeltlichen Vermittlung.

Aus einer methodischen Sicht kann nach Ansicht der Autoren die hier zur Diskussion gestellte, relativ zeitnahe, Abschätzung der Wirkungen eines Programms der aktiven Arbeitsmarktpolitik mit Individualdaten wertvolle Informationen für eine rationale Weiterentwicklung der Arbeitsmarktpolitik bereitstellen. Jedoch darf der zeitliche und sonstige Aufwand für die Beschaffung der Daten und deren ökonometrischer Auswertung nicht unterschätzt werden. Für jedes Arbeitsmarktprogramm muss aufgrund seiner Besonderheiten idealerweise ein maßgeschneidertes Untersuchungsdesign entwickelt werden. Zudem müssen die Programmwirkungen abgewartet werden, bevor sie analysiert werden können. In unserem Beispiel des VGS sind dies immerhin 12 Monate. Weitere Verzögerungen ergeben sich aus dem Zeitraum, in dem die ökonometrische Analyse erfolgt, und dem Zeitraum, in dem deren Ergebnisse mit den verantwortlichen Gremien diskutiert wird. Daraus können sich Anforderungen an weitere Analysen ergeben, usw.

Diese Verzögerungen können dazu führen, dass die Relevanz der gefundenen Programmwirkungen von politischen Entscheidungsträgern in Frage gestellt werden kann. Denn in der Zwischenzeit können sich die politischen und wirtschaftlichen Rahmenbedingungen so verschoben haben, dass sich auch die Programmwirkungen wieder geändert haben könnten. Ohne eine Rückkopplung mittels mikroökonometrischer Evidenz besteht für die aktive Arbeitsmarktpolitik jedoch die Gefahr, nicht aus der Sackgasse herauszufinden. 


\section{Literaturverzeichnis}

Almus, M., Egeln, J., Lechner, M., Pfeiffer, F. und H. Spengler (1998), Die gemeinnützige Arbeitnehmerüberlassung in Rheinland-Pfalz - eine ökonometrische Analyse des Wiedereingliederungserfolges, MittAB 31, 558-575.

Beckmann, M., Deimel, J. und B. Schauenburg (2004), Vermittlungsgutscheine als neues Instrument der Arbeitsmarktpolitik: eine erste Analyse, Perspektiven der Wirtschaftspolitik 5 (2), 127-138.

Bundesagentur für Arbeit (2004), Arbeitsmarkt 2004, Amtliche Nachrichten der Bundesagentur für Arbeit, 53. Jg., Sondernummer.

Deutscher Bundestag (2002), Begründung zum „Gesetz zur Vereinfachung der Wahl der Arbeitnehmervertreter in den Aufsichtsrat“ (BT-Drucksache 12/8546).

Franz, W. und F. Pfeiffer (2005), A Note on Labor Contracts and Wage Rigidities: An Empirical Investigation Using Survey Data, Applied Economics Quarterly 51 (2), 219-228.

Heckman, J. J., R. J. LaLonde und J. A. Smith (1999), The Economics and Econometrics of Active Labour Market Programs, in: Ashenfelter, O. und D. Card (Hrsg.), Handbook of Labor Economics Vol. 3a, Elsevier, Amsterdam, 1865-2097.

Heinze, A., F. Pfeiffer, A. Spermann, H. Winterhager and A. Wuppermann (2005), Vermittlungsgutscheine, Teil I - Datenstruktur und deskriptive Analysen, IABForschungsbericht 1/2005.

Hujer, R. Caliendo, M. und Thomsen, S. (2004), New Evidence on the Effects of Job Creation Schemes in Germany - A Matching Approach with Threefold Heterogeneity, Research in Economics 58 (4), 257-302.

Hujer, R., P. Rodrigues und C. Zeiss (2006), Makroökonometrische Analyse der Effekte von Vermittlungsgutscheinen, in: Kruppe, T. (2006.).

Hummel, E., P. Jacobebbinghaus, A. Kohlmann, M. Oertel, C. Wübbeke und M. Ziegerer (2005): Stichprobe der Integrierten Erwerbsbiografien - IEBS 1.0, Handbuch-Version 1.0.0, FDZ Datenreport Nr. 06.

Konle-Seidl, R. (2002), Steigerung von Effizienz und Reputation in der Arbeitsvermittlung, IAB-Werkstattbericht 15.

Konle-Seidl, R. (2005), New Delivery Forms of Employment Services in Germany: A Mixed Public-Private Model?, in Sol, E. und M. Westerveld (Hrsg.), Contractualism in Employment Services, Kluwer, Amsterdam, 187-208.

Konle-Seidl, R. and U. Walwei (2002), Wandel der Arbeitsvermittlung durch Deregulierung: Mehr Reputation durch Marktöffnung?, In: G. Kleinhenz, IAB Kompendium Arbeitsmarkt- und Berufsforschung, Beiträge zur Arbeitsmarkt- und Berufsforschung 250, 171-185.

Kruppe, T. (Hrsg.) (2006), Private Vermittlung als Unterstützung: Eine Evaluation von Vermittlungsgutscheinen und Beauftragungen Dritter, BeitrAB 301, IAB, Nürnberg.

Kruppe, T. und M. Oertel, (2003), Von Verwaltungsdaten zu Forschungsdaten - die Individualdaten für die Evaluation des ESF-BA-Programms 2000 bis 2006, IABWerkstattbericht 10.

Lechner, M. (1999), Earnings and Employment Effects of Continuous Off-the-Job Training in East Germany After Unification, Journal of Business \& Economic Statistics 17 (1), 74-90. 
Lechner, M. (2002), Some Practical Issues in the Evaluation of Heterogeneous Labour Market Programmes by Matching Methods, Journal of the Royal Statistical Society Association 165, 59-82.

Lechner, M. und F. Pfeiffer (Hrsg.) (2001), Econometric Evaluation of Labor Market Policies, Physica, Heidelberg.

Lechner, M., Pfeiffer, F., Spengler H. und M. Almus (2001), The Impact of Non-profit Temping Agencies on Individual Labor Market Success, in: Lechner, M. und F. Pfeiffer (Hrsg.), Econometric Evaluation of Labor Market Policies, Physica, Heidelberg, 43-58.

Pfeiffer, F. (2003), Lohnrigiditäten im gemischten Lohnbildungssystem, Nomos, BadenBaden.

Pfeiffer, F. und W. Pohlmeier (Hrsg.) (1998): Qualifikation, Weiterbildung und Arbeitsmarkterfolg, Nomos, Baden-Baden.

Pries, M. und R. Rogerson (2005), Hiring Policies, Labor Market Institutions, and Labor Market Flows, Journal of Political Economy 113 (4), 811-839.

Rosenbaum, P. R. und D. B. Rubin (1983), The Central Role of the Propensity Score in Observational Studies for Causal Effects, Biometrika 70 (1), 1-55.

Rubin, D.B. (1974), Estimating Causal Effects of Treatments in Randomized and Nonrandomized Studies, Journal of Educational Psychology 66, 688-701.

Ullrich, B. (2004), Der Vermittlungsgutschein als Weg aus der Arbeitslosigkeit? Ein theoriegeleitetes Evaluationskonzept der arbeitsmarktpolitischen Maßnahme, Universität Lüneburg, Arbeitsbericht Nr. A325.

Van der Linden, B. (2005), Equilibrium Evaluation of Active Labor Market Programmes Enhancing Matching Effectiveness), IZA Discussion Paper 1526.

Wilke, R. und H. Winterhager (2004), Datenquellen als Voraussetzung für Evaluationsforschung, in: Hagen, T. und A. Spermann: Hartz-Gesetze - Methodische Ansätze zu einer Evaluierung, Nomos, Baden-Baden, 33-43.

Winterhager, H. (2006), Contracting Out Placement Services - A Microeconometric Evaluation for Germany, Mimeo, ZEW Mannheim. 


\section{Anhang}

\section{Schätzung des Propensity Scores}

Der Propensity Score wurde mit einem Probit-Modell geschätzt. Die Schätzung wurde so spezifiziert, dass die erklärenden Variablen möglichst gut geeignet für das nachfolgende Matching sind (s.u.). Die Schätzung erfolgt getrennt für Ost- und Westdeutschland sowie für Gutscheine, die in unterschiedlichen Quartalen ausgegeben wurden. In Tabelle A1 werden die Ergebnisse der beiden Schätzungen für das 3. Quartal 2003 vorgestellt. Die Ergebnisse für die anderen Quartale sind qualitativ vergleichbar.

Tabelle A1: Probitschätzungen des Propensity Scores (Vermittlungsgutschein gegenüber Nichtbezug), getrennt für Ost und West, Ausgaben im 3. Quartal 2003

\begin{tabular}{|c|c|c|c|c|}
\hline & \multicolumn{2}{|c|}{ West } & \multicolumn{2}{|l|}{ Ost } \\
\hline \multirow[t]{2}{*}{ Pseudo R-squared } & \multicolumn{2}{|c|}{0,1198} & \multicolumn{2}{|l|}{0,0961} \\
\hline & $\begin{array}{l}\text { Koeffizient } \\
\text { (marginaler } \\
\text { Effekt) }\end{array}$ & & $\begin{array}{l}\text { Koeffizient } \\
\text { (marginaler } \\
\text { Effekt) }\end{array}$ & \\
\hline \multicolumn{5}{|l|}{ Allgemeine sozioökonomische Merkmale } \\
\hline \multicolumn{5}{|l|}{ Splinefunktion } \\
\hline Alter 0 & 0,00067 & 3,18 & $-0,00323$ & $-7,32$ \\
\hline Alter 25 & $-0,00005$ & $-0,28$ & $-0,00065$ & $-1,76$ \\
\hline Alter 30 & 0,00001 & 0,04 & 0,00039 & 1,16 \\
\hline Alter 35 & 0,00030 & 2,25 & 0,00055 & 1,97 \\
\hline Alter 40 & 0,00018 & 1,38 & $-0,00072$ & $-2,77$ \\
\hline Alter 45 & $-0,00103$ & $-7,40$ & $-0,00200$ & $-7,52$ \\
\hline Alter 50 & $-0,00248$ & $-15,68$ & $-0,00470$ & $-14,80$ \\
\hline Alter 55 & $-0,00807$ & $-23,90$ & $-0,01591$ & $-23,37$ \\
\hline Alter 60 & $-0,00410$ & $-3,52$ & $-0,01068$ & $-3,19$ \\
\hline Ausländer & $-0,00260$ & $-6,50$ & $-0,01668$ & $-7,29$ \\
\hline Frau & $-0,00160$ & $-3,63$ & $-0,00717$ & $-6,42$ \\
\hline Allein lebend & Referenz & & & \\
\hline In Partnerschaft nicht verheiratet & 0,00237 & 3,09 & 0,01086 & 8,40 \\
\hline allein erziehend & 0,00023 & 0,16 & $-0,00576$ & $-1,92$ \\
\hline verheiratet & 0,00311 & 7,83 & 0,01465 & 16,60 \\
\hline Frau * (in Partnerschaft nicht verheiratet) & $-0,00231$ & $-2,02$ & $-0,00882$ & $-4,80$ \\
\hline Frau * (allein erziehend) & 0,00123 & 0,75 & 0,00831 & 2,38 \\
\hline
\end{tabular}




\begin{tabular}{|c|c|c|c|c|}
\hline Frau * (verheiratet) & $-0,00972$ & $-17,51$ & $-0,01794$ & $-14,81$ \\
\hline Jüngstes Kind 0-3 Jahre & 0,00834 & 0,40 & $-0,02558$ & $-0,93$ \\
\hline Jüngstes Kind 3-7 Jahre & $-0,00079$ & $-1,42$ & $-0,00620$ & $-5,38$ \\
\hline Jüngstes Kind 7-14 Jahre & 0,00009 & 0,18 & $-0,00184$ & $-2,01$ \\
\hline Alter 0 * Jüngstes Kind 0-3 Jahre & $-0,00033$ & $-0,42$ & 0,00115 & 0,80 \\
\hline Alter 25 * Jüngstes Kind 0-3 Jahre & $-0,00045$ & $-0,87$ & $-0,00028$ & $-0,27$ \\
\hline Alter 30 * Jüngstes Kind 0-3 Jahre & 0,00051 & 1,06 & $-0,00074$ & $-0,68$ \\
\hline Alter 35 * Jüngstes Kind 0-3 Jahre & 0,00012 & 0,22 & 0,00016 & 0,12 \\
\hline Alter 40 * Jüngstes Kind 0-3 Jahre & $-0,00050$ & $-0,59$ & $-0,00243$ & $-1,04$ \\
\hline Alter 45 * Jüngstes Kind 0-3 Jahre & $-0,00077$ & $-0,48$ & 0,00616 & 1,53 \\
\hline Alter 50 * Jüngstes Kind 0-3 Jahre & 0,00191 & 0,66 & 0,00514 & 0,69 \\
\hline Alter 55 * Jüngstes Kind 0-3 Jahre & $-0,00511$ & $-0,50$ & $-0,00211$ & $-0,07$ \\
\hline Alter 60 * Jüngstes Kind 0-3 Jahre & 0,02262 & 0,74 & & \\
\hline Teilzeit * Jüngstes Kind 0-3 Jahre & $-0,00165$ & $-0,91$ & 0,00275 & 0,48 \\
\hline \multicolumn{5}{|l|}{ Gesundheitszustand: } \\
\hline Keine gesundheitlichen Einschränkungen & Referenz & & & \\
\hline Behinderungsgrad (BG) 80\% & $-0,01503$ & $-12,92$ & $-0,03217$ & $-8,68$ \\
\hline BG $50 \%$ & $-0,00972$ & $-15,50$ & $-0,02368$ & $-14,20$ \\
\hline BG 30\%, nicht gleichgestellt & $-0,00543$ & $-7,36$ & $-0,00945$ & $-4,68$ \\
\hline BG 30\% gleichgestellt & $-0,00198$ & $-1,38$ & $-0,00577$ & $-2,32$ \\
\hline Sonst. gesundheitliche Einschränkungen & $-0,00424$ & $-11,36$ & $-0,00790$ & $-9,70$ \\
\hline \multicolumn{5}{|l|}{ Qualifikation } \\
\hline \multicolumn{5}{|l|}{ Schulabschluss: } \\
\hline Ohne Abschluss & Referenz & & & \\
\hline Hauptschulabschluss & 0,00166 & 3,73 & 0,00222 & 1,92 \\
\hline Mittlere Reife & 0,00455 & 8,31 & 0,00163 & 1,38 \\
\hline Fachhochschulreife & 0,00282 & 3,56 & $-0,00676$ & $-2,90$ \\
\hline Abitur & 0,00212 & 2,88 & $-0,00360$ & $-1,94$ \\
\hline \multicolumn{5}{|l|}{ Berufliche Ausbildung: } \\
\hline Keine Ausbildung & Referenz & & & \\
\hline Betriebliche Ausbildung & 0,00774 & 9,07 & 0,01975 & 11,21 \\
\hline Außerbetriebliche Ausbildung & 0,01903 & 10,07 & 0,03290 & 11,63 \\
\hline Berufsfachschule, Fachschule & 0,01301 & 6,64 & 0,03398 & 6,78 \\
\hline Universität & 0,00789 & 3,57 & 0,00210 & 0,31 \\
\hline Fachhochschule & 0,00930 & 3,20 & 0,02243 & 2,57 \\
\hline \multicolumn{5}{|l|}{$\begin{array}{l}\text { Qualifikation nach Einschätzung des Agentur- } \\
\text { mitarbeiters: }\end{array}$} \\
\hline Kräfte mit und ohne Fachkenntnisse & Referenz & & & \\
\hline Fachkräfte & 0,00559 & 15,17 & 0,01139 & 17,45 \\
\hline Kräfte mit Fachschulniveau & 0,01026 & 10,50 & 0,01513 & 7,21 \\
\hline Kräfte mit Fachhochschulniveau & 0,00604 & 4,61 & 0,01709 & 5,95 \\
\hline Kräfte mit Hochschulniveau & $-0,00145$ & $-1,21$ & 0,00498 & 1,70 \\
\hline Spitzenkräfte & $-0,00414$ & $-0,85$ & 0,02749 & 1,48 \\
\hline Sonstige Kräfte & 0,00554 & 4,91 & 0,02570 & 10,03 \\
\hline
\end{tabular}


Berufserfahrung im gewünschten Beruf (Ein-

schätzung des Agenturmitarbeiters)

\begin{tabular}{rrrr}
0,00332 & 4,97 & 0,01085 & 6,17 \\
$-0,00221$ & $-2,58$ & $-0,01291$ & $-6,32$ \\
& & & \\
$-0,00166$ & $-0,99$ & $-0,01086$ & $-3,93$ \\
$-0,00447$ & $-2,69$ & $-0,01395$ & $-3,49$ \\
$-0,00323$ & $-1,73$ & 0,00709 & 1,01 \\
$-0,00333$ & $-1,35$ & $-0,00777$ & $-1,05$ \\
\hline$-0,00130$ & $-4,90$ & 0,00214 & 3,75 \\
\hline
\end{tabular}

Berufserfahrung * Betriebliche Ausbildung

Berufserfahrung * Außerbetriebliche Aus-

bildung

Berufserfahrung * Berufsfachschule

Berufserfahrung * Universität

Referenz

Präferierte Arbeitszeit:

Nur Vollzeit

Nur Teilzeit

Zukünftige Arbeitszeit egal

$-0,01072$

$-0,00105$

$-0,02047 \quad-10,42$

Vollzeit, auch Schicht

$0,01377 \quad 9,50$

0,00235

1,85

Sonstige Arbeitszeit

0,00349

0,58

$0,00478 \quad 3,36$

Letzter Kontakt mit Arbeitsmarkt

Tagesentgelt bei letzter Beschäftigung (Dummy, wenn zutreffend)

$\begin{array}{llll}0,00100 & 2,43 & -0,00472 & -3,89\end{array}$

Tagesentgelt bei letzter Beschäftigung ( $€$ )

$\begin{array}{llll}0,00001 & 1,32 & 0,00029 & 7,98\end{array}$

Tagesentgelt bei letzter Beschäftigung (€),

Quadrat

$\begin{array}{llll}0,00000 & -0,90 & 0,00000 & -5,07\end{array}$

Grund für Beendigung des letzten Arbeitsverhältnisses:

Kündigung durch Arbeitgeber

$\begin{array}{llll}0,00305 & 7,55 & 0,00366 & 3,74\end{array}$

Befristeter Vertrag

0,00271

5,47

$-0,00465$

$-4,83$

Sonstiger Grund

Referenz

Frühere Abmeldungen aus Arbeitslosigkeit:

Krankmeldungen, Dummy

$\begin{array}{llll}0,00081 & 2,07 & 0,00317 & 4,26\end{array}$

Krankmeldungen, Anzahl

$-0,00043 \quad-4,19$

$-0,00042$

$-1,89$

Fehlende Verfügbarkeit, Dummy

$0,00158 \quad 2,79$

0,00297

1,76

Fehlende Verfügbarkeit, Anzahl

$-0,00162 \quad-5,40$

$-0,00748$

$-7,00$

Sperrzeiten, Dummy

$0,00188 \quad 0,87$

0,01660

1,98

Sperrzeiten, Anzahl

$-0,00180 \quad-0,97$

$-0,00802$

$-1,15$

Säumniszeiten, Dummy

$0,00201 \quad 1,07$

$-0,00308$

$-0,72$

Säumniszeiten, Anzahl

$-0,00428$

$-2,72$

$-0,00329$

$-0,87$

Leistungsbezug pro Tag (€)

Leistungsbezug, Höhe

$\begin{array}{llll}0,00056 & 11,14 & 0,00088 & 7,02\end{array}$

Leistungsbezug, Höhe, Quadrat

$-0,00001 \quad-11,50$

$-0,00002 \quad-5,96$

Berücksichtigung saisonaler Effekte

Ausgabe im August

0,00066

2,09

0,00468

7,27

Ausgabe im September

$0,00380 \quad 12,41$

0,00365

5,79

Die folgenden unabhängigen Variablen wurden aus Platzgründen nicht dargestellt: Dummyvariablen zur Beschreibung der langfristigen Arbeitsmarkthistorie der Individuen und Splinefunktion für die bisherige Dauer des augenblicklichen Arbeitslosigkeitsspells (121 Variablen), Dummyvariablen für den Agenturbezirk (146 in Westdeutschland und 34 in Ostdeutschland) 


\section{Balancing Tests}

Durch das Matching sollen die beobachtbaren Merkmale der VGS-Bezieher und der Kontrollgruppe aus Nichtbeziehern möglichst gut angeglichen werden. Diese Angleichung kann durch Balancing Tests überprüft werden. Ein häufig angewendeter Test ist die standardisierte Differenz, die von Rosenbaum und Rubin (1985) eingeführt wurde. Diese ist vor bzw. nach Matching definiert als

$$
\operatorname{SDIFF}_{\text {vor }}=100 \frac{\bar{X}_{1}-\bar{X}_{0}}{\sqrt{\left(V_{1}\left(X_{1}\right)+V_{0}\left(X_{0}\right)\right) / 2}} \quad \operatorname{SDIFF}_{\text {nach }}=100 \frac{\bar{X}_{1 M}-\bar{X}_{0 M}}{\sqrt{\left(V_{1}\left(X_{1}\right)+V_{0}\left(X_{0}\right)\right) / 2}} .
$$

Dabei sind $\bar{X}_{1}$ und $\bar{X}_{0}$ die Mittelwerte der betrachteten Kovariaten in den gesamten (ungematchten) Teilnehmer- und Nichtteilnehmer-Samples, $\bar{X}_{1 M}$ und $\bar{X}_{0 M}$ diejenigen in den gematchten Samples. $V_{1}\left(X_{1}\right)$ und $V_{0}\left(X_{0}\right)$ sind die Varianzen in den gesamten (ungematchten) Teilnehmer- und Nichtteilnehmer-Samples. Es wird somit in beiden Fällen mit den Stichprobenvarianzen vor Matching normiert, so dass die standardisierten Differenzen vor und nach der Kontrollgruppenbildung vergleichbar sind. Problematisch ist an diesem Test, dass es kein formales Kriterium dafür gibt, welche Größe der standardisierten Differenz noch akzeptabel ist. Rosenbaum und Rubin (1985) bezeichnen einen Wert von 20 als groß (,large“).

Die Spezifikation der im vorherigen Abschnitt dargestellten Schätzung des Propensity Scores wurde so gewählt, dass diese Tests eine möglichst gute Ausbalancierung anzeigen. Die Ergebnisse werden beispielhaft für das dritte Quartal dargestellt (Tabelle X3), wobei nur wichtige ausgewählte Variablen angegeben werden. Die übrigen Ergebnisse sind qualitativ vergleichbar. 
Tabelle A2: Standardisierte Differenzen für ausgewählte Kovariate, vor und nach Matching (Ausgaben von Vermittlungsgutscheinen im 3. Quartal 2003)

\begin{tabular}{|c|c|c|c|c|}
\hline & \multicolumn{2}{|c|}{ West } & \multicolumn{2}{|c|}{ Ost } \\
\hline & Vor & Nach & Vor & Nach \\
\hline & & & & \\
\hline Alter & 25,84 & 0,28 & 29,30 & 0,93 \\
\hline Ausländische Nationalität & 13,61 & 0,07 & 2,65 & 0,46 \\
\hline Frau & 9,05 & 0,00 & 21,53 & 0,00 \\
\hline In Partnerschaft nicht verheiratet & 3,58 & 0,30 & 3,55 & 0,47 \\
\hline allein erziehend & 3,08 & 0,88 & 3,56 & 0,93 \\
\hline verheiratet & 12,16 & 0,82 & 6,30 & 0,57 \\
\hline Jüngstes Kind 0-3 Jahre & 2,51 & 0,13 & 4,82 & 1,44 \\
\hline Jüngstes Kind 3-7 Jahre & 2,53 & 0,10 & 0,32 & 0,29 \\
\hline Jüngstes Kind 7-14 Jahre & 0,52 & 0,20 & 0,65 & 0,40 \\
\hline Behinderungsgrad (BG) 80\% & 7,04 & 0,12 & 4,06 & 0,19 \\
\hline BG 50\% & 11,83 & 0,58 & 8,42 & 0,42 \\
\hline BG 30\%, nicht gleichgestellt & 8,24 & 0,31 & 3,99 & 0,06 \\
\hline BG 30\%, gleichgestellt & 1,71 & 0,25 & 2,43 & 0,10 \\
\hline $\begin{array}{l}\text { Sonstige gesundheitliche Ein- } \\
\text { schränkungen }\end{array}$ & 11,93 & 0,48 & 14,56 & 0,17 \\
\hline Hauptschulabschluss & 12,28 & 0,83 & 10,71 & 0,46 \\
\hline Mittlere Reife & 21,05 & 0,19 & 14,24 & 0,50 \\
\hline Fachhochschulreife & 5,48 & 0,82 & 2,69 & 0,27 \\
\hline Abitur & 4,90 & 0,86 & 2,50 & 0,31 \\
\hline betriebliche Ausbildung & 17,39 & 0,50 & 8,68 & 1,14 \\
\hline Außerbetriebliche Ausbildung & 10,06 & 0,38 & 10,60 & 0,96 \\
\hline Berufsfachschule, Fachschule & 4,04 & 0,07 & 0,54 & 0,66 \\
\hline Universität & 1,20 & 0,21 & 0,69 & 0,14 \\
\hline Fachhochschule & 3,63 & 1,05 & 3,04 & 0,36 \\
\hline Fachkräfte & 21,66 & 0,38 & 22,40 & 1,06 \\
\hline Kräfte mit Fachschulniveau & 5,46 & 0,80 & 2,65 & 0,83 \\
\hline Kräfte mit Fachhochschulniveau & 3,81 & 0,41 & 3,05 & 0,17 \\
\hline Kräfte mit Hochschulniveau & 0,83 & 0,73 & 0,57 & 0,14 \\
\hline Spitzenkräfte & 0,70 & 0,06 & 0,31 & 0,44 \\
\hline Sonstige Kräfte & 1,07 & 0,24 & 0,98 & 0,12 \\
\hline $\begin{array}{l}\text { Arbeitslosigkeitsdauer bis zum } \\
\text { (hyp.) Ausgabezeitpunkt }\end{array}$ & 24,71 & 0,02 & 33,13 & 0,10 \\
\hline $\begin{array}{l}\text { Tagesentgelt bei letzter Beschäfti- } \\
\text { gung }(€)\end{array}$ & 7,40 & 0,44 & 9,29 & 0,15 \\
\hline Leistungsbezug, Höhe & 12,09 & 0,38 & 21,45 & 0,74 \\
\hline Ausgabe im August & 1,89 & 0,00 & 2,03 & 0,00 \\
\hline Ausgabe im September & 3,95 & 0,00 & 0,75 & 0,00 \\
\hline
\end{tabular}




\section{Ergebnisse des Matching}

Im Folgenden werden die Ergebnisse des Nearest Neighbor Propensity Score Matching mit Zurücklegen dargestellt. Für die Berechnung des Standardfehlers des ATT werden unabhängige Beobachtungen, feste Gewichte und Homoskedastizität der Zielvariable innerhalb der Treatment Gruppe angenommen (siehe Lechner, 2001). Die Varianz wird dadurch beeinflusst, dass der Propensity Score geschätzt wurde. Um die Standardfehler konsistent zu schätzen, müsste daher Bootstrapping angewendet werden (vgl. Lechner, 2002), was für den hier verwendeten großen Datensatz sehr zeitaufwändig wäre. Lechner (2002) zeigt in einer angewandten Studie für die Schweiz, dass der Unterschied zwischen der gebootstrappten Varianz und derjenigen, die sich in der folgenden Gleichung ergibt, gering ist. Diese Gleichung nimmt an, dass die Varianz nicht dadurch beeinflusst wird, dass der Propensity Score geschätzt wurde:

$\operatorname{Var}(A T T)=\frac{1}{N_{1}} \operatorname{Var}\left(Y_{1} \mid D=1\right)+\frac{\sum_{j=1}^{N_{0}} \omega_{j}^{2}}{\left(N_{1}\right)^{2}} \operatorname{Var}\left(Y_{1} \mid D=0\right)$.

wobei $N_{1}$ die Anzahl der VGS-Bezieher ist und $\omega_{j}$ das Gewicht der Beobachtung j im Matching. Diese Gleichung wurde hier verwendet, um die Standardfehler des ATT zu berechnen. In den Tabellen A3 und A4 finden sich die Anteile in regulärer Beschäftigung in Teilnehmer- und Kontrollgruppe, der geschätzte ATT als Differenz dieser beiden Größen sowie die Standardfehler. 
Tabelle A3: Geschätzte ATT für die VGS-Bezieher in Ostdeutschland: Anteil in regulärer Beschäftigung; für VGS-Bezieher Anteil der ausgegebenen VGS, die eingelöst werden (Ausgaben Mai 2003 bis Dezember 2003)

\begin{tabular}{lrrrrr}
$\begin{array}{l}\text { Dauer nach Ana- } \\
\text { lysezeitpunkt } \\
\text { (Monate) }\end{array}$ & $\begin{array}{l}\text { VGS- } \\
\text { Bezieher }\end{array}$ & \multicolumn{1}{l}{$\begin{array}{l}\text { Kontroll- } \\
\text { gruppe }\end{array}$} & Differenz & Std.fehler & t-Wert \\
\hline Beobachtungen & 141.097 & 141.097 & & \\
1 & $7,33 \%$ & $4,26 \%$ & $3,07 \%$ & $0,08 \%$ & 38,4 \\
2 & $11,19 \%$ & $6,97 \%$ & $4,22 \%$ & $0,10 \%$ & 42,2 \\
3 & $13,30 \%$ & $8,77 \%$ & $4,53 \%$ & $0,11 \%$ & 41,2 \\
4 & $14,39 \%$ & $9,95 \%$ & $4,44 \%$ & $0,12 \%$ & 37,0 \\
5 & $15,21 \%$ & $10,83 \%$ & $4,38 \%$ & $0,12 \%$ & 36,5 \\
6 & $15,80 \%$ & $11,55 \%$ & $4,25 \%$ & $0,12 \%$ & 35,4 \\
7 & $16,39 \%$ & $12,04 \%$ & $4,35 \%$ & $0,13 \%$ & 33,5 \\
8 & $17,20 \%$ & $12,73 \%$ & $4,48 \%$ & $0,13 \%$ & 34,5 \\
9 & $18,49 \%$ & $13,60 \%$ & $4,90 \%$ & $0,13 \%$ & 37,7 \\
10 & $19,57 \%$ & $14,39 \%$ & $5,17 \%$ & $0,14 \%$ & 36,9 \\
11 & $20,81 \%$ & $15,27 \%$ & $5,54 \%$ & $0,14 \%$ & 39,6 \\
12 & $21,83 \%$ & $15,97 \%$ & $5,86 \%$ & $0,14 \%$ & 41,9 \\
\hline \hline
\end{tabular}

Tabelle A4: Geschätzte ATT für die VGS-Bezieher in Westdeutschland: Anteil in regulärer Beschäftigung; für VGS-Bezieher Anteil der ausgegebenen VGS, die eingelöst werden (Ausgaben Mai 2003 bis Dezember 2003)

\begin{tabular}{lrrrrr}
$\begin{array}{l}\text { Dauer nach Ana- } \\
\text { lysezeitpunkt } \\
\text { (Monate) }\end{array}$ & \multicolumn{1}{l}{$\begin{array}{l}\text { VGS- } \\
\text { Bezieher }\end{array}$} & \multicolumn{1}{l}{$\begin{array}{l}\text { Kontroll- } \\
\text { gruppe }\end{array}$} & Differenz & Std.fehler & t-Wert \\
\hline Beobachtungen & 191.222 & 191.222 & & \\
1 & $6,42 \%$ & $4,70 \%$ & $1,72 \%$ & $0,07 \%$ & 24,6 \\
2 & $10,62 \%$ & $7,80 \%$ & $2,82 \%$ & $0,09 \%$ & 31,3 \\
3 & $13,59 \%$ & $10,10 \%$ & $3,49 \%$ & $0,10 \%$ & 34,9 \\
4 & $15,63 \%$ & $11,84 \%$ & $3,79 \%$ & $0,11 \%$ & 34,5 \\
5 & $17,27 \%$ & $13,23 \%$ & $4,04 \%$ & $0,12 \%$ & 33,7 \\
6 & $18,65 \%$ & $14,44 \%$ & $4,20 \%$ & $0,12 \%$ & 35,0 \\
7 & $19,76 \%$ & $15,35 \%$ & $4,41 \%$ & $0,12 \%$ & 36,8 \\
8 & $20,92 \%$ & $16,25 \%$ & $4,67 \%$ & $0,12 \%$ & 38,9 \\
9 & $22,12 \%$ & $17,27 \%$ & $4,85 \%$ & $0,13 \%$ & 37,3 \\
10 & $23,06 \%$ & $18,12 \%$ & $4,95 \%$ & $0,13 \%$ & 38,1 \\
11 & $24,05 \%$ & $18,90 \%$ & $5,15 \%$ & $0,13 \%$ & 39,6 \\
12 & $24,80 \%$ & $19,46 \%$ & $5,34 \%$ & $0,13 \%$ & 41,1 \\
\hline \hline
\end{tabular}

